\title{
Transport coefficients in the Polyakov quark meson coupling model: A relaxation time approximation
}

\author{
Aman Abhishek ${ }^{1,2, *}$ and Hiranmaya Mishra ${ }^{1, \dagger}$ \\ ${ }^{1}$ Theory Division, Physical Research Laboratory, Navrangpura, Ahmedabad 380 009, India \\ ${ }^{2}$ Indian Institute of Technology Gandhinagar, Gandhinagar 382355, Gujarat, India \\ Sabyasachi Ghosh ${ }^{3}$ \\ ${ }^{3}$ Department of Physics, University of Calcutta, 92, A.P.C. Road, Kolkata 700009, India
}

(Received 4 October 2017; published 12 January 2018)

\begin{abstract}
We compute the transport coefficients, namely, the coefficients of shear and bulk viscosities, as well as thermal conductivity for hot and dense matter. The calculations are performed within the Polyakov quark meson model. The estimation of the transport coefficients is made using the Boltzmann kinetic equation within the relaxation time approximation. The energy-dependent relaxation time is estimated from mesonmeson scattering, quark-meson scattering, and quark-quark scattering within the model. In our calculations, the shear viscosity to entropy ratio and the coefficient of thermal conductivity show a minimum at the critical temperature, while the ratio of bulk viscosity to entropy density exhibits a peak at this transition point. The effect of confinement modeled through a Polyakov loop potential plays an important role both below and above the critical temperature.
\end{abstract}

DOI: 10.1103/PhysRevD.97.014005

\section{INTRODUCTION}

Transport coefficients of matter under extreme conditions of temperature, density, or external fields are interesting for several reasons. In the context of relativistic heavy ion collisions, these properties enter as dissipative coefficients in the hydrodynamic evolution of the quark gluon plasma that is produced following the collision [1-5]. Indeed, an extremely low value of the shear viscosity-to-entropy ratio $(\eta / s)$ is needed to successfully describe the collective dynamics of the quark gluon matter at high temperature and vanishing chemical potential to explain the elliptic flow data [6,7]. At intermediate densities, near the chiral phase transition, which is being probed at the Facility for antiproton and Ion Research (FAIR) program at Geselleschaft fuer Schwerionenforschung (GSI)-[8] and the Nuclotronbased Ion Collider fAcility (NICA) program at Joint Institute for Nuclear Research(JINR)-[9] motivates us to understand the behavior of transport coefficients at finite chemical potential and temperature. Further, in the low-temperature and high-density regime, the matter could be in one of the

\footnotetext{
*aman@prl.res.in

hm@prl.res.in
}

Published by the American Physical Society under the terms of the Creative Commons Attribution 4.0 International license. Further distribution of this work must maintain attribution to the author(s) and the published article's title, journal citation, and DOI. Funded by SCOAP. possible types of color superconducting phases of which the transport properties also need to be understood $[10,11]$. The cooling of neutron stars at short time scales constrains the thermal conductivity $[12,13]$, while the cooling through neutrino emission on much larger time scales constrains the phase of the matter in the interior of the compact star $[14,15]$. Further, the observable regarding the viscosity of the such matter is the r-mode instability. In the absence of viscous damping, the fluid in the rotating star becomes unstable to a mode that is coupled to gravity and radiates away the angular momentum of the star $[16,17]$. Apart from the wide variety of applications of the transport coefficients of strongly interacting matter, their temperature and chemical potential dependence may also be indicative of a phase transition [18].

Transport coefficients for QCD matter in principle can be calculated using Kubo formulation [19]. However, QCD is strongly interacting for both at energies accessible in heavy ion collision experiments as well as for the densities expected to be there in the core of the neutron stars making the perturbative estimations unreliable. Calculations using lattice QCD simulations at finite chemical potential are also challenging and are limited only to the equilibrium thermodynamic properties at small chemical potentials.

The understanding of the elliptic flow in relativistic heavy ion collisions using hydrodynamics with a low $(\eta / s)$ and its connection to the conjectured lower bound $(\eta / s>1 / 4 \pi)$ using ADS/CFT correspondence [20] stimulated extensive investigation of this ratio for QCD matter. These have been studied using perturbative QCD [21], 
transport simulations of the Boltzmann equation [22,23], relaxation time approximation for solving the Boltzmann equations [24-27], and lattice simulation of QCD [28]. Most of these calculations have been performed at vanishing baryon density. The general variation of this ratio with temperature in most of these studies shows a minimum at the transition temperature. The numerical value of $\eta$ at the minimum, however, differs by an order of magnitude. For example, near transition temperature, Refs. [29-33] have predicted $\eta$ of order $0.001 \mathrm{GeV}^{3}, \eta=0.002-0.003 \mathrm{GeV}^{3}$, while Ref. [34] predicts a value of $\eta \simeq 0.4 \mathrm{GeV}^{3}$. Further, the behavior of $\eta / s$ shows a monotonic decrease in the Nambu-Jona-Lasinio (NJL) model in Ref. [35].

The bulk viscosity coefficient $\zeta$ has also been estimated in various effective models as well as in lattice QCD. The rise of the bulk viscosity coefficient near the transition temperature has been observed in these effective models such as chiral perturbation theory [36], quasiparticle models [37], the linear sigma model [38], and the Nambu-Jona-Lasinio model [24,25]. Large bulk viscosity of matter produced in relativistic heavy ion collisions can give rise to different interesting phenomenon such as cavitation where pressure vanishes and hydrodynamic description of evolution becomes invalid [39]. Here, again, the numerical values of the bulk viscosity coefficients vary widely from $10^{-5}$ [40] to $10^{-2} \mathrm{GeV}^{3}$ [24].

The other transport coefficient that is important at finite baryon density is the coefficient of thermal conductivity $\lambda$ [41-43]. The effects of thermal conductivity in relativistic hydrodynamics have been discussed recently in Refs. $[43,44]$. This coefficient has been evaluated in various effective models like the Nambu-Jona-Lasinio model using the Green-Kubo approach [45], relaxation time approximation [25], and the instanton liquid model [46]. The results, however, vary over a wide range of values, with $\lambda=0.008 \mathrm{GeV}^{-2}$ as in Ref. [31] to $\lambda \sim 10 \mathrm{GeV}^{-2}$ as in Ref. [35] for a range of temperatures $(0.12 \mathrm{GeV}<\mathrm{T}<$ $0.17 \mathrm{GeV}$ ), which has been nicely tabulated in Ref. [47].

We shall attempt here to estimate these transport coefficients within an effective model of strong interaction, the Polyakov loop extended quark meson (PQM) model. It has become quite popular during last few years due to its close relationship with the linear sigma model that captures the chiral symmetry breaking aspect while being in agreement with the lattice QCD results for thermodynamics at vanishing baryon density. The physics of confinement is taken care of at least partially by coupling the quark field to the Polyakov loops so that quark excitations are suppressed below the transition temperature. Let us note that the transport coefficients like bulk viscosity apart from the distribution functions also depend upon the bulk thermodynamic quantities like the velocity of sound. We wish to explore the effects of such nonperturbative properties on the transport coefficients.

The transport coefficients are evaluated within the relaxation time approximation of the Boltzmann equation.
The relaxation time is calculated by evaluating the scattering rates of the particles in the model, namely, the quarks and pion and sigma mesons, with their respective mediumdependent masses. The scattering processes considered here are meson scatterings as considered in Ref. [38], quark scattering through meson exchanges as in Refs. [24,25,35] and quark-meson scattering. As we shall see in the following, each of these processes brings out distinct features for the transport coefficients. We would like to mention here that these coefficients have also been estimated using Kubo formulation through one-loop self-energies for quarks and mesons in a separate work [48].

We organize the present investigation as follows. In the following section, we discuss the two-flavor PQM model thermodynamics. The reason is that the expressions for transport coefficients involve meson masses which are medium dependent. Further, some transport coefficients like the bulk viscosity involves bulk thermodynamical properties such as energy density, pressure and the velocity of sound. As the order parameters for chiral and confinement-deconfinement transitions are coupled, this leads to nontrivial relations for derivatives of the thermodynamic potential with respect to external parameters like chemical potential or temperature as the mean fields themselves are also medium dependent. Furthermore, the implicit dependence of these mean fields/order parameters are calculated here analytically to avoid possible numerical errors. In Sec. III, we give the expressions for the transport coefficients in terms of relaxation time and estimate them to finally give the results for these coefficients. We also compare them with the same obtained with alternate approaches like the NJL model so that the effects of the confinement-deconfinement transition modeled through the Polyakov loop potential are explicitly seen. Finally, we summarize and draw the conclusions of the present investigation in Sec. IV.

\section{THERMODYNAMICS OF PQM MODEL}

We shall adopt here an effective model that captures two important features of QCD, namely, chiral symmetry breaking and its restoration at high temperature and/ densities as well as the confinement-deconfinement transitions. Two such effective models have become popular recently-the Polyakov loop extended Nambu-JonaLasinio model and the PQM. These models are extensions, respectively, of the NJL model and linear sigma model that captures various aspects of chiral symmetry breaking pattern of strong interaction physics. Explicitly, the Lagrangian of the PQM model is given by [49-53]

$$
\begin{aligned}
\mathcal{L}= & \bar{\psi}\left(i \gamma^{\mu} D_{\mu}-m-g_{\sigma}\left(\sigma+i \gamma_{5} \boldsymbol{\tau} \cdot \boldsymbol{\pi}\right)\right) \psi \\
& +\frac{1}{2}\left[\partial_{\mu} \sigma \partial^{\mu} \sigma+\partial_{\mu} \pi \partial^{\mu} \pi\right]-U_{\chi}(\sigma, \pi)-U_{P}(\phi, \bar{\phi}) .
\end{aligned}
$$

In the above, the first term is the kinetic and interaction term for the quark doublet $\psi=(u, d)$ interacting with the scalar $(\sigma)$ and the isovector pseudoscalar pion $(\boldsymbol{\pi})$ field. The 
scalar field $\sigma$ and the pion field $\pi$ together form an SU(2) isovector field. The quark field is also coupled to a spatially constant temporal gauge field $A_{0}$ through the covariant derivative $D_{\mu}=\partial_{\mu}-i e A_{\mu} ; A_{\mu}=\delta_{\mu 0} A_{\mu}$.

The mesonic potential $U_{\chi}(\sigma, \pi)$ essentially describes the chiral symmetry breaking pattern in strong interaction and is given by

$$
U_{\chi}(\sigma, \pi)=\frac{\lambda}{4}\left(\sigma^{2}+\pi^{2}-v^{2}\right)^{2}-c \sigma .
$$

The last term in the Lagrangian in Eq. (1) is responsible for including the physics of color confinement in terms of a potential energy for the expectation value of the Polyakov loop $\phi$ and $\bar{\phi}$, which are defined in terms of the Polyakov loop operator, which is a Wilson loop in the temporal direction,

$$
\mathcal{P}=P \exp \left(i \int_{0}^{\beta} d x_{0} A_{0}\left(x_{0}, \mathbf{x}\right)\right) .
$$

In the Polyakov gauge, $A_{0}$ is time independent and is in the Cartan subalgebra, i.e., $A_{0}^{a}=A_{0}^{3} \lambda_{3}+A_{0}^{8} \lambda_{8}$. One can perform the integration over the time variable trivially as path ordering becomes irrelevant so that $\mathcal{P}(\mathbf{x})=\exp \left(\beta A_{0}\right)$. The Polyakov loop variable $\phi$ and its Hermitian conjugate $\bar{\phi}$ are defined as

$$
\phi(\mathbf{x})=\frac{1}{N_{c}} \operatorname{Tr} \mathcal{P}(\mathbf{x}) \quad \bar{\phi}(\mathbf{x})=\frac{1}{N_{c}} \mathcal{P}^{\dagger}(\mathbf{x}) .
$$

In the limit of heavy quark mass, the confining phase is center symmetric, and therefore $\langle\phi\rangle=0$, while for the deconfined phase, $\langle\phi\rangle \neq 0$. Finite quark masses break this symmetry explicitly. The explicit form of the potential $U_{p}(\phi, \bar{\phi})$ is not known from first principle calculations. The common strategy is to choose a functional form of the potential that reproduces the pure gauge lattice simulation thermodynamic results. Several forms of this potential have been suggested in the literature. We shall use here the polynomial parametrization [49]

$U_{P}(\phi, \bar{\phi})=T^{4}\left[-\frac{b_{2}(T)}{2} \bar{\phi} \phi-\frac{b_{3}}{2}\left(\phi^{3}+\bar{\phi}^{3}\right)+\frac{b_{4}}{4}(\bar{\phi} \phi)^{2}\right]$

with the temperature-dependent coefficient $b_{2}$ given as

$$
b_{2}(T)=a_{0}+a_{1}\left(\frac{T_{0}}{T}\right)+a_{2}\left(\frac{T_{0}}{T}\right)^{2}+a_{3}\left(\frac{T_{0}}{T}\right)^{3} .
$$

The numerical values of the parameters are

$$
\begin{aligned}
& a_{0}=6.75, \quad a_{1}=-1.95, \\
& a_{2}=2.625, \quad a_{3}=-7.44 \\
& b_{3}=0.75, \quad b_{4}=7.5 .
\end{aligned}
$$

The parameter $T_{0}$ corresponds to the transition temperature of Yang-Mills theory. However, for the full dynamical QCD, there is a flavor dependence on $T_{0}\left(N_{f}\right)$. For two flavors, we take it to be $T_{0}(2)=192 \mathrm{MeV}$ as in Ref. [49].

The Lagrangian in Eq. (1) is invariant under $S U(2)_{L} \times$ $S U(2)_{R}$ transformation when the explicit symmetry breaking term $c \sigma$ vanishes in the potential $U_{\chi}$ in Eq. (2). The parameters of the potential $U_{\chi}$ are chosen such that the chiral symmetry is spontaneously broken in the vacuum. The expectation values of the meson fields in vacuum are $\langle\sigma\rangle=f_{\pi}$ and $\langle\boldsymbol{\pi}\rangle=0$. Here, $f_{\pi}=93 \mathrm{MeV}$ is the pion decay constant. The coefficient of the symmetry breaking linear term is decided from the partial conservation of the axial vector current as $c=f_{\pi} m_{\pi}^{2}, m_{\pi}=138 \mathrm{MeV}$, being the pion mass. Then, minimizing the potential, one has $v^{2}=f_{\pi}^{2}-m_{\pi}^{2} / \lambda$. The quartic coupling for the meson $\lambda$ is determined from the mass of the sigma meson given as $m_{\sigma}^{2}=m_{\pi}^{2}+2 \lambda f_{\pi}^{2}$. In the present work, we take $m_{\sigma}=$ $600 \mathrm{MeV}$, which gives $\lambda=19.7$. The coupling $g_{\sigma}$ is fixed here from the constituent quark mass in vacuum $M_{q}=g_{q} f_{\pi}$, which has to be about one-third of nucleon mass that leads to $g_{\sigma}=3.3$ [54].

To calculate the bulk thermodynamical properties of the system, we use a mean field approximation for the meson and the Polyakov fields while retaining the quantum and thermal fluctuations of the quark fields. The thermodynamic potential can then be written as

$$
\Omega(T, \mu)=\Omega_{\bar{q} q}+U_{\chi}+U_{P}(\phi, \bar{\phi}) .
$$

The fermionic part of the thermodynamic potential is given as

$$
\Omega_{\bar{q} q}=-2 N_{f} T \int \frac{d^{3} p}{(2 \pi)^{3}}\left[\ln \left(1+3\left(\phi+\bar{\phi} e^{-\beta \omega_{-}}\right) e^{-\beta \omega_{-}}+e^{-3 \beta \omega_{-}}\right)+\ln \left(1+3\left(\phi+\bar{\phi} e^{-\beta \omega_{+}}\right) e^{-\beta \omega_{+}}+e^{-3 \beta \omega_{+}}\right)\right]
$$

modulo a divergent vacuum part. In the above, $\omega_{\mp}=$ $E_{p} \mp \mu$, with the single particle quark/antiquark energy $E_{p}=\sqrt{\mathbf{p}^{2}+M^{2}}$. The constituent quark/antiquark mass is defined to be

$$
M^{2}=g_{\sigma}^{2}\left(\sigma^{2}+\pi^{2}\right)
$$

The divergent vacuum part arises from the negative energy states of the Dirac sea. Using standard renormalization, it can be partly absorbed in the coupling $\lambda$ and $v^{2}$. 
However, a logarithmic correction from the renormalization scale remains, and we neglect it in the calculations that follow [54].

The mean fields are obtained by minimizing $\Omega$ with respect to $\sigma, \phi, \bar{\phi}$, and $\pi$. Extremizing the effective potential with respect to the $\sigma$ field leads to

$$
\lambda\left(\sigma^{2}+\pi^{2}-v^{2}\right)-c+g_{\sigma} \rho_{s}=0,
$$

where the scalar density $\rho_{s}=-\langle\bar{\psi} \psi\rangle$ is given by

$$
\rho_{s}=6 N_{f} g_{\sigma} \sigma \int \frac{d \mathbf{p}}{(2 \pi)^{3}} \frac{1}{E_{P}}\left[f_{-}(\mathbf{p})+f_{+}(\mathbf{p})\right] .
$$

In the above, $f_{\mp}(\mathbf{p})$ are the distribution functions for the quarks and antiquarks given as

$$
f_{-}(\mathbf{p})=\frac{\phi e^{-\beta \omega_{-}}+2 \bar{\phi} e^{-2 \beta \omega_{-}}+e^{-3 \beta \omega_{-}}}{1+3 \phi e^{-\beta \omega_{-}}+3 \bar{\phi} e^{-2 \beta \omega_{-}}+e^{-3 \beta \omega_{-}}}
$$

and

$$
f_{+}(\mathbf{p})=\frac{\bar{\phi} e^{-\beta \omega_{+}}+2 \phi e^{-2 \beta \omega_{+}}+e^{-3 \beta \omega_{+}}}{1+3 \bar{\phi} e^{-\beta \omega_{+}}+3 \phi e^{-2 \beta \omega_{+}}+e^{-3 \beta \omega_{+}}} .
$$

The condition $\frac{\partial \Omega}{\partial \phi}=0$ leads to

$$
T^{4}\left[-\frac{b_{2}}{2} \bar{\phi}-\frac{b_{3}}{2} \phi^{2}+\frac{b_{4}}{2} \bar{\phi} \phi \bar{\phi}\right]+I_{\phi}=0,
$$

where

$$
\begin{aligned}
I_{\phi}= & \frac{\partial \Omega_{\bar{q} q}}{\partial \phi}=-6 N_{f} T \int \frac{d \mathbf{p}}{(2 \pi)^{3}} \\
& \times\left[\frac{e^{-\beta \omega_{-}}}{1+3 \phi e^{-\beta \omega_{-}}+3 \bar{\phi} e^{-2 \beta \omega_{-}}+e^{-3 \beta \omega_{-}}}\right. \\
& \left.+\frac{e^{-2 \beta \omega_{+}}}{1+3 \bar{\phi} e^{-\beta \omega_{+}}+3 \phi e^{-2 \beta \omega_{+}}+e^{-3 \beta \omega_{+}}}\right] .
\end{aligned}
$$

Similarly, $\frac{\partial \Omega}{\partial \bar{\phi}}=0$ leads to

$$
T^{4}\left[-\frac{b_{2}}{2} \phi-\frac{b_{3}}{2} \bar{\phi}^{2}+\frac{b_{4}}{2} \bar{\phi} \phi^{2}\right]+I_{\bar{\phi}}=0
$$

with

$$
\begin{aligned}
I_{\bar{\phi}}= & \frac{\partial \Omega_{\bar{q} q}}{\partial \bar{\phi}}=-6 N_{f} T \int \frac{d \mathbf{p}}{(2 \pi)^{3}} \\
& \times\left[\frac{e^{-2 \beta \omega_{-}}}{1+3 \phi e^{-\beta \omega_{-}}+3 \bar{\phi} e^{-2 \beta \omega_{-}}+e^{-3 \beta \omega_{-}}}\right. \\
& \left.+\frac{e^{-\beta \omega_{+}}}{1+3 \phi e^{-\beta \omega_{+}}+3 \bar{\phi} e^{-2 \beta \omega_{+}}+e^{-3 \beta \omega_{+}}}\right] .
\end{aligned}
$$

Finally, minimization of the effective potential with respect to $\pi$ fields leads to

$$
\frac{\partial \Omega}{\partial \pi}=\lambda\left(\sigma^{2}+\pi^{2}-v^{2}\right) \pi+g \rho_{p s}=0,
$$

where the pseudoscalar density can be expressed as

$$
\begin{aligned}
\rho_{p s} & =\left\langle\bar{q} l \gamma_{5} \tau q\right\rangle \\
& =6 N_{f} g_{\sigma} \pi \int \frac{d \mathbf{p}}{(2 \pi)^{3}} \frac{1}{E_{P}}\left[f_{-}(\mathbf{p})+f_{+}(\mathbf{p})\right] .
\end{aligned}
$$

The $\sigma$ and $\pi$ masses are determined by the curvature of $\Omega$ at the global minimum

$$
M_{\sigma}^{2}=\frac{\partial^{2} \Omega}{\partial \sigma^{2}}, \quad M_{\pi_{i}}^{2}=\frac{\partial^{2} \Omega}{\partial \pi_{i}^{2}} .
$$

These equations lead to the masses for the $\sigma$ and pions given as

$$
\begin{gathered}
M_{\sigma}^{2}=m_{\pi}^{2}+\lambda\left(3 \sigma^{2}-f_{\pi}^{2}\right)+g_{\sigma}^{2} \frac{\partial \rho_{s}}{\partial \sigma} \\
M_{\pi}^{2}=m_{\pi}^{2}+\lambda\left(\sigma^{2}-f_{\pi}^{2}\right)+g_{\sigma}^{2} \frac{\partial \rho_{p s}}{\partial \pi} .
\end{gathered}
$$

Explicitly, using Eq. (13),

$$
\begin{aligned}
\frac{\partial \rho_{s}}{\partial \sigma}= & \frac{6}{\pi^{2}} \int d p p^{2} \\
& \times\left[\frac{g_{\sigma} p^{2}}{E(\mathbf{p})^{3}}\left(f_{-}(\mathbf{p})+f_{+}(\mathbf{p})\right)+\frac{M}{E(\mathbf{p})}\left(\frac{\partial f_{-}}{\partial \sigma}+\frac{\partial f_{+}}{\partial \sigma}\right)\right] .
\end{aligned}
$$

The derivatives of the distribution functions with respect to the scalar field $\sigma$ are given as

$$
\frac{\partial f_{-}(\mathbf{p})}{\partial \sigma}=\frac{\beta g_{\sigma}^{2} \sigma}{E(\mathbf{p})}\left[3 f_{-}^{2}-\frac{3 e^{-3 \beta \omega_{-}}+4 \bar{\phi} e^{-2 \beta \omega_{-}}+\phi e^{-\beta \omega_{-}}}{1+3 \phi e^{-\beta \omega_{-}}+3 \bar{\phi} e^{-2 \beta \omega_{-}}+e^{-3 \beta \omega_{-}}}\right]
$$

and

$$
\frac{\partial f_{+}}{\partial \sigma}=\frac{\beta g_{\sigma}^{2} \sigma}{E(\mathbf{p})}\left[3 f_{+}^{2}-\frac{3 e^{-3 \beta \omega_{+}}+4 \phi e^{-2 \beta \omega_{+}}+\bar{\phi} e^{-\beta \omega_{+}}}{1+3 \bar{\phi} e^{-\beta \omega_{+}}+3 \phi e^{-2 \beta \omega_{+}}+e^{-3 \beta \omega_{+}}}\right] .
$$

Similarly, using Eq. (21),

$$
\frac{\partial \rho_{p s}}{\partial \pi}=\frac{6}{\pi^{2}} \int d p \frac{p^{2}}{E(\mathbf{p})}\left[f_{-}(\mathbf{p})+f_{+}(\mathbf{p})\right] .
$$




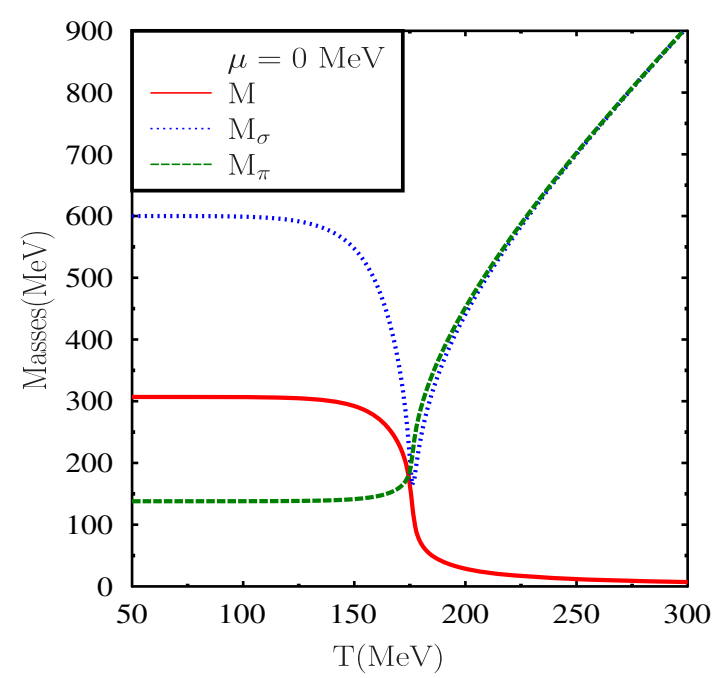

(a)

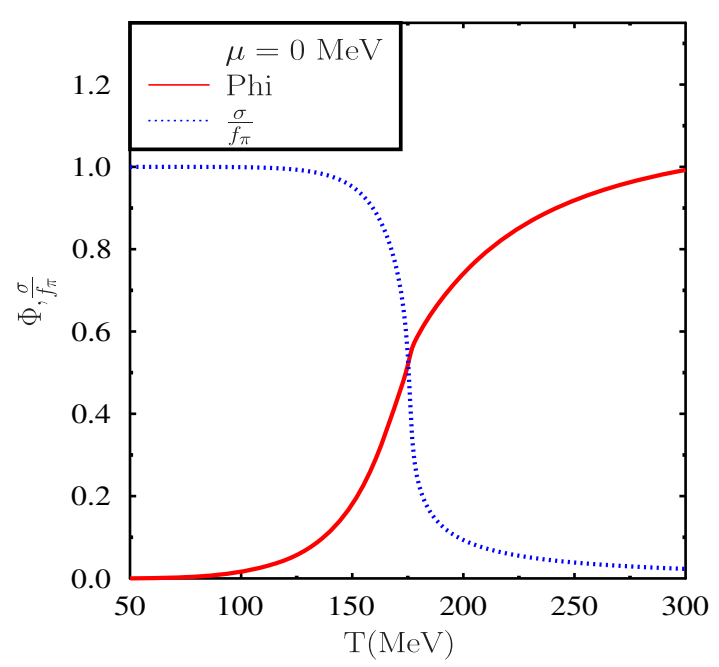

(b)

FIG. 1. (a) Temperature dependence of the masses of constituent quarks $(M)$ and pions $\left(M_{\pi}\right)$ and sigma mesons $\left(M_{\sigma}\right)$ and $(\mathrm{b})$ the order parameters $\sigma$ and $\phi$ as a function of temperature for $\mu=0 \mathrm{MeV}$.

In the above, we have set the expectation value of the pion field to be zero, i.e., $\boldsymbol{\pi}=0$, so that the constituent quark mass is $M^{2}=g_{\sigma}^{2} \sigma^{2}$.

The net quark density is given by

$$
n=-\frac{\partial \Omega}{\partial \mu}=\frac{6}{\pi^{2}} \int p^{2} d p\left[f_{-}(\mathbf{p})-f_{+}(\mathbf{p})\right]
$$

The energy density $\epsilon=\Omega-T \partial \Omega / \partial T+\mu \rho_{q}$ is given by

$$
\begin{aligned}
\epsilon= & \frac{6}{\pi^{2}} \int p^{2} d p E(\mathbf{p})\left(f_{-}(\mathbf{p})+f_{+}(\mathbf{p})\right)+U_{\chi}-3 U_{P}(\phi, \bar{\phi}) \\
& +\frac{T^{5}}{2} \frac{d b_{2}(T)}{d T} \bar{\phi} \phi .
\end{aligned}
$$

In Fig. 1(a), we have plotted the constituent quark mass and the meson masses as given in Eqs. (23) and (24) as a function of temperature for vanishing baryon density. In the chirally broken phase, the pion mass, being the mass of an approximate Goldstone mode, is protected and varies weakly with temperature. On the other hand, the mass of $\sigma, M_{\sigma}$, which is approximately twice the constituent quark mass, $M$, drops significantly near the crossover temperature. At high temperature, being chiral partners, the masses of the $\sigma$ and $\pi$ mesons become degenerate and increase linearly with temperature. In Fig. 1(b), we have plotted the order parameters $\sigma$ and $\phi$ as a function of temperature for the vanishing quark chemical potential. We also note that for $\mu=0$ the order parameters $\phi$ and $\bar{\phi}$ are the same. Because of the approximate chiral symmetry, the chiral order parameter decreases with temperatures to small values but never vanishes. The Polyakov loop parameter, on the other hand, grows from $\phi=0$ at zero temperature to about $\phi=1$ at high temperatures. We might mention here that at very high temperature the value of the Polyakov loop parameter exceeds unity, the value in the infinite quark mass limit.

Next, in Fig. 2, we show the dependence of the trace anomaly $(\epsilon-3 p) / T^{4}$ on temperature. The conformal symmetry is broken maximally at the critical temperature. Further, finite chemical potential enhances this breaking as it breaks scale symmetry explicitly. As we shall see later, this will have its implication on the bulk viscosity coefficients.

Next, to discuss critical behavior as well as to calculate different thermodynamic quantities, one has to take derivatives of the thermodynamic potential with respect to the mean fields as well as the parameters like temperature and the chemical potential. Vanishing of the first-order

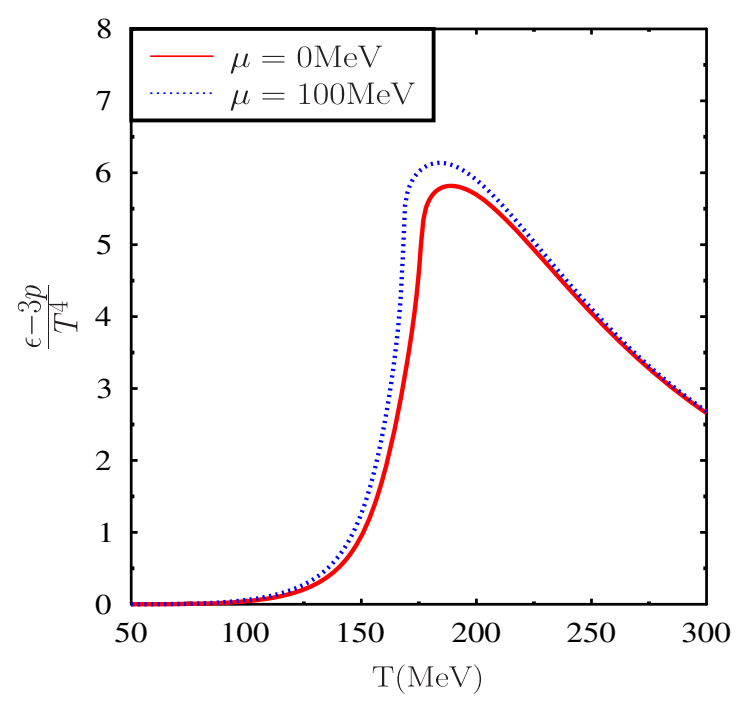

FIG. 2. Temperature dependence of the scaled trace anomaly $\frac{\epsilon-3 p}{T^{4}}$. 


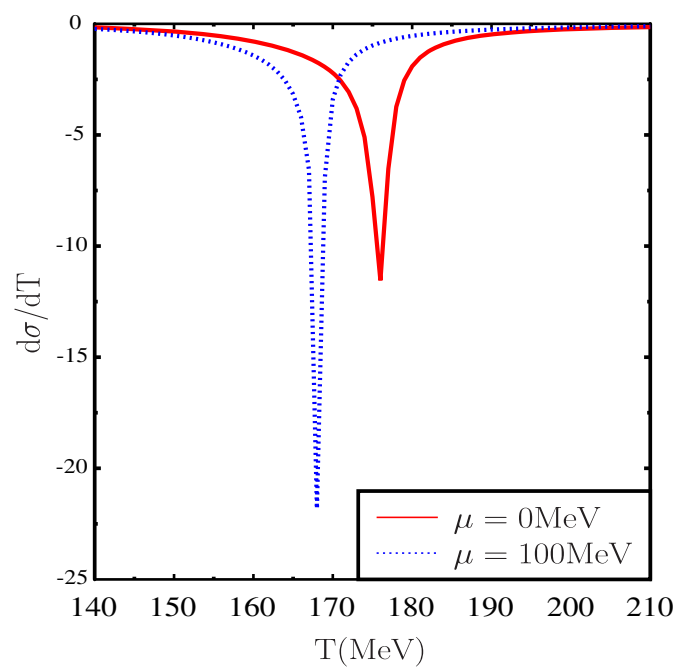

(a)

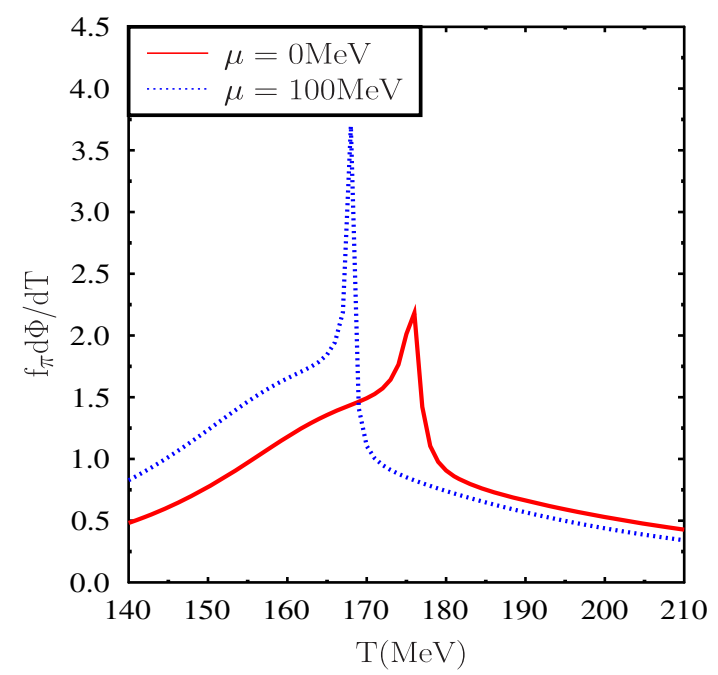

(b)

FIG. 3. (a) Temperature derivative of the chiral order parameter $\left(\frac{d \sigma}{d T}\right)$ and (b) Polyakov loop parameter $\left(\frac{d \phi}{d T}\right)$ as a function of temperature.

derivatives of the thermodynamic potential with respect to the order parameters leads to the values of the order parameters satisfying the coupled gap equations as shown. However, to calculate many different thermodynamic quantities, one also has to take into account the implicit dependence of the order parameters on temperature as well as chemical potential. One can do a numerical differentiation of the order parameters after solving for them from the gap equation. However, this can be numerically less accurate, particularly for the higher-order derivatives. We shall use here a semianalytic approach to calculate the implicit contributions to the extent of taking the differentiation of the expressions analytically [55]. Only the values of the final expressions so obtained are computed numerically. For example, to calculate the derivative of the order parameter $X,(X=\sigma, \phi, \bar{\phi})$ with respect to temperature is given by the equation

$$
\begin{aligned}
& \frac{\partial}{\partial T}\left(\frac{\partial \Omega}{\partial X}\right)+\frac{\partial}{\partial \sigma}\left(\frac{\partial \Omega}{\partial X}\right) \frac{d \sigma}{d T}+\frac{\partial}{\partial \phi}\left(\frac{\partial \Omega}{\partial X}\right) \frac{d \phi}{d T} \\
& \quad+\frac{\partial}{\partial \bar{\phi}}\left(\frac{\partial \Omega}{\partial X}\right) \frac{d \bar{\phi}}{d T}=0 .
\end{aligned}
$$

Thus, we have a matrix equation of the type $\mathbf{A} \cdot \mathbf{Y}=\mathbf{B}$, where $\mathbf{A}$ is the coefficient matrix of the variables $\mathbf{Y}=\left(\frac{\mathbf{d} \sigma}{\mathbf{d T}}, \frac{\mathbf{d} \phi}{\mathbf{d T}}, \frac{\mathbf{d} \bar{\phi}}{\mathbf{d T}}\right)^{\mathbf{T}}$, and $\mathbf{B}$ is the matrix of derivatives of the thermodynamic potential involving explicit dependence on temperature, i.e., $\mathbf{B}=\left(-\frac{\partial}{\partial \mathbf{T}}\left(-\frac{\partial \Omega}{\partial \sigma},-\frac{\partial \Omega}{\partial \phi},-\frac{\partial \Omega}{\partial \bar{\phi}}\right) \mathbf{T}\right)$. These matrix equations can be solved using Cramer's rule. The coefficient matrix $\mathbf{A}$ is given by

$$
\mathbf{A}=\left[\begin{array}{lll}
\Omega_{\sigma \sigma} & \Omega_{\sigma \phi} & \Omega_{\sigma \bar{\phi}} \\
\Omega_{\phi \sigma} & \Omega_{\phi \phi} & \Omega_{\phi \bar{\phi}} \\
\Omega_{\bar{\phi} \sigma} & \Omega_{\bar{\phi} \phi} & \Omega_{\bar{\phi} \bar{\phi}}
\end{array}\right]
$$

with $\Omega_{a b}=\frac{\partial^{2} \Omega}{\partial a \partial b}$, where $a, b$ stand for $\sigma, \phi$ and $\bar{\phi}$. Similarly, to calculate the derivatives with respect to chemical potential, the coefficient matrix $\mathbf{A}$ remains the same, while the matrix $\mathbf{B}$ will involve derivatives of the thermodynamic potential involving explicit dependence on the chemical potential.

Solving Eq. (31) this way, we have plotted the derivatives of the order parameters in Fig. 3. The critical temperature is defined by the position of the peaks of these derivatives of the order parameters. At zero chemical potential, this occurs at $T_{C} \simeq 176 \mathrm{MeV}$. Let us note that at $T_{C}$ the quark mass is $m_{q}=g_{\sigma} \sigma=134 \mathrm{MeV}$, while the Polyakov loop variable $\phi \sim=0.5$. Thus, at the critical temperature, the effect of interaction is significant. As the chemical potential for the quarks increases, the critical temperature decreases. With finite chemical potential, the peaks also become sharper, and at higher chemical potential, the transition becomes a first-order one. The critical point within this model occurs at $\left(T_{c}, \mu_{c}\right)=$ $(155,163) \mathrm{MeV}$.

The other thermodynamic quantity that enters into the transport coefficient calculation is the velocity of sound. The same at constant density is defined as

$$
c_{s}^{2}=\left(-\frac{\partial P}{\partial \epsilon}\right)_{n}=\frac{s \chi_{\mu \mu}-\rho \chi_{\mu T}}{T\left(\chi_{T T} \chi_{\mu \mu}-\chi_{\mu T}^{2}\right)},
$$

where $P$, the pressure, is the negative of the thermodynamic potential given in Eq. (9). Further, $s=-\frac{\partial \Omega}{\partial T}$ is the entropy density, and the susceptibilities are defined as $\chi_{x y}=-\frac{\partial^{2} \Omega}{\partial x \partial y}$. The velocity of sound shows a minimum near the crossover temperature as may be seen in Fig. 4. Within the model, at low temperature when the constituent quarks start contributing to the pressure, their contribution to the energy 


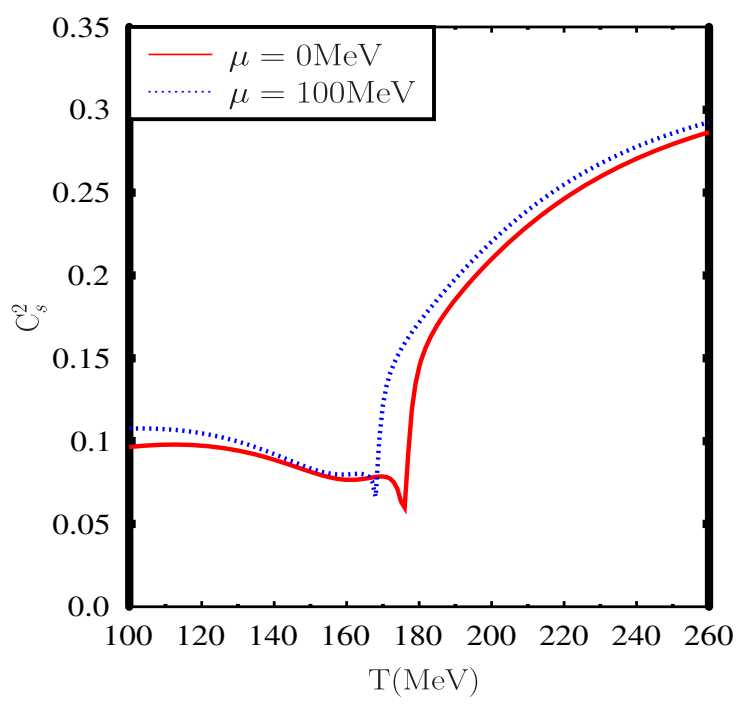

FIG. 4. Temperature dependence of the velocity of sound at constant density.

density is significant compared to their contribution to the pressure, leading to decreasing behavior of the velocity of sound until the crossover temperature, beyond which it increases as the quarks become light and approach the massless limit of $c_{s}^{2}=\frac{1}{3}$. Such a dip in the velocity of sound is also observed in lattice simulation [56]. As we shall observe later, this behavior will have important consequences for the behavior of bulk viscosity as a function of temperature. We might mention here that such a dip for the sound velocity was not observed for two-flavor NJL [25]. For the linear sigma model calculations, such a dip was observed only for a large sigma meson mass [38].

\section{TRANSPORT COEFFICIENTS IN RELAXATION TIME APPROXIMATION}

We shall attempt here to estimate the transport coefficients in the relaxation time approximation where the particle masses are medium dependent. Such attempts were made earlier for the $\sigma$ model [38] as well as in the NJL model to compute the shear and bulk viscosity coefficients. Such an approach was also made to estimate the viscosity coefficients of pure gluon matter [57]. In all these attempts, the expressions for the viscosity coefficients were derived for vanishing chemical potential. Several attempts were made to estimate these coefficients with finite chemical potential with different Ansätze. These expressions were put on firmer ground by deriving the expressions when there are mean fields and mediumdependent masses in a quasiparticle picture [58]. The resulting expressions for the transport coefficients were manifestly positive definite as they should be. These expressions were derived explicitly for the NJL model [25]. We use the same expressions here for the transport coefficients. The shear viscosity coefficient is given by

$$
\eta=\frac{1}{15 T} \sum_{a} \int \frac{d \mathbf{p}}{(2 \pi)^{3}} \frac{p_{a}^{4}}{E_{a}^{2}} \tau\left(E_{a}\right) f_{a}^{0}\left(1 \pm f_{a}^{0}\right),
$$

where the sum is over all the different species contributing to the viscosity coefficients including the antiparticles and $\tau^{a}$ is the energy-dependent relaxation time that we define in the following subsection. The coefficient of bulk viscosity is given by

$$
\begin{aligned}
\zeta= & \frac{1}{9 T} \sum_{a} \int \frac{d \mathbf{p}}{(2 \pi)^{3}} \frac{\tau^{a}}{E_{a}{ }^{2}} f_{a}{ }^{0}\left(1 \pm f_{a}{ }^{0}\right)\left[\mathbf{p}^{2}\left(1-3 v_{n}^{2}\right)\right. \\
& -3 v_{n}^{2}\left(M^{2}-T M \frac{d M}{d T}-\mu M \frac{d M}{d \mu}\right) \\
& \left.+3\left(\frac{\partial P}{\partial n}\right)_{\epsilon}\left(M \frac{d M}{d \mu}-E_{a} t^{a}\right)\right]^{2} .
\end{aligned}
$$

The thermal conductivity, on the other hand, is given by

$$
\begin{aligned}
\lambda= & \left(\frac{w}{n T}\right)^{2} \sum_{a} \int \frac{d \mathbf{p}}{(2 \pi)^{3}} \frac{\mathbf{p}^{2}}{3 E_{a}^{2}} \tau_{a}\left(E_{a}\right)\left(t_{a}-\frac{n E_{a}}{w}\right)^{2} \\
& \times f_{a}^{0}\left(1 \pm f_{a}^{0}\right) .
\end{aligned}
$$

In the above expressions, $f_{a}^{0}$ is the equilibrium fermion/ boson distribution functions depending upon the statistics with $\left(1 \pm f_{a}^{0}\right)$ being the Bose enhancement/Fermi suppression factors and $t_{a}=+1,-1$, and 0 for the quark, antiquark, and meson, respectively. Further, $c_{s}^{2}=\left(\frac{\partial p}{\partial \epsilon}\right)_{n}$ is the velocity of sound at constant density, and $w=\epsilon+p$ is the enthalpy density.

\section{A. Relaxation time estimation-Meson scatterings}

As may be noted, the expressions for the transport coefficients as in Eqs. (34), (35), and (36) depend not only on bulk thermodynamic properties like energy density, pressure, and velocity of sound but also on the energydependent relaxation time $\tau(E)$. In the following, we shall first estimate the relaxation times involving meson exchanges similar to Ref. [38].

Using the Lagrangian Eq. (1), we calculate the relaxation time in the PQM model by taking into account the following scattering amplitudes with the corresponding matrix elements being given as

$$
\begin{aligned}
M_{\sigma+\sigma \rightarrow \sigma+\sigma}= & -6 \lambda-36 \lambda^{2} f_{\pi}^{2} \\
& \times\left(\frac{1}{s-m_{\sigma}^{2}}+\frac{1}{t-m_{\pi}^{2}}+\frac{1}{u-m_{\pi}^{2}}\right) \\
M_{\pi+\sigma \rightarrow \pi+\sigma}= & -2 \lambda-4 \lambda^{2} f_{\pi}^{2} \\
& \times\left(\frac{3}{t-m_{\sigma}^{2}}+\frac{1}{u-m_{\pi}^{2}}+\frac{1}{s-m_{\pi}^{2}}\right)
\end{aligned}
$$




$$
\begin{aligned}
M_{\pi+\pi \rightarrow \pi+\pi}= & -2 \lambda\left(\frac{s-m_{\pi}^{2}}{s-m_{\sigma}^{2}} \delta_{\mathrm{ab}} \delta_{\mathrm{cd}}+\frac{t-m_{\pi}^{2}}{t-m_{\sigma}^{2}} \delta_{\mathrm{ac}} \delta_{\mathrm{bd}}\right. \\
& \left.+\frac{u-m_{\pi}^{2}}{u-m_{\sigma}^{2}} \delta_{\mathrm{ad}} \delta_{\mathrm{bc}}\right) \\
M_{\pi+\pi \rightarrow \sigma+\sigma}= & -6 \lambda-4 \lambda^{2} f_{\pi}^{2} \\
& \times\left(\frac{3}{s-m_{\sigma}^{2}}+\frac{1}{t-m_{\pi}^{2}}+\frac{1}{u-m_{\pi}^{2}}\right) .
\end{aligned}
$$

The terms involving the propagators yield divergent integrals due to the poles in the $\mathrm{s}$ and $\mathrm{u}$ channels, which is known in the literature [38]. To regulate these integrals, one can include a width for the mesons as evaluated in the next subsection [Eq. (54)]. However, such a substitution violates crossing symmetry. Further, these terms are generated from the three-point vertices, which are not taken into account in the mean field approximation used in solving the gap equations and the resulting equation of state. Hence, to be consistent with the equation of state while maintaining crossing symmetry for the scattering amplitudes, we approximate the above scattering amplitudes by their limits when $s, t$, and $u$ are taken to be infinity and the scattering amplitudes reduce to constants [38]. Thus, the scattering amplitudes essentially reduce to constants. This allows us to compare our results with the earlier work of Ref. [38] and study the effect of the Polyakov loop and quarks within a similar approximation.

The energy-dependent interaction frequency $\omega_{a}\left(E_{a}\right)$ for the particle species $a$ arising from a scattering process $a, b \rightarrow c, d$, which is also the inverse of the energydependent relaxation time $\tau\left(E_{a}\right)$, is given by, with $d \Gamma_{i}=$ $\frac{d \mathbf{p}_{i}}{2 E_{i}(\mathbf{p})(2 \pi)^{3}}[25]$

$$
\omega\left(E_{a}\right) \equiv \tau\left(E_{a}\right)^{-1}=\sum_{b} \int d \Gamma_{b} f_{b}^{0} W_{a b}(s) .
$$

In the above, the summation is over all the particles except the species $a$ with $a, b$ as the initial state.

The quantity $W_{a b}$ is dimensionless and Lorentz invariant, and depends only on the Mandelstam variable $s$ and is given by

$$
\begin{aligned}
W_{a b}(s)= & \frac{1}{1+\delta_{a b}} \int d \Gamma_{c} d \Gamma_{d}(2 \pi)^{4} \delta^{4}\left(p_{a}+p_{b}-p_{c}-p_{d}\right) \\
& \times|M|^{2}\left(1+f_{c}\right)\left(1+f_{d}\right) .
\end{aligned}
$$

In the above, we have included the Bose enhancement factors for the meson scattering. The quantity $W_{a b}(s)$ is related to the cross section by noting that, with $t$ as the Mandelstam variable $t=\left(p_{a}-p_{c}\right)^{2}$,

$$
\frac{d \sigma}{d t}=\frac{1}{64 \pi s} \frac{1}{p_{a b}^{2}}|M|^{2},
$$

where $p_{a b}(s)=1 /(2 \sqrt{s}) \sqrt{\lambda\left(s, m_{a}^{2}, m_{b}^{2}\right)}$, and the kinematic function $\lambda(x, y, z)=x^{2}+y^{2}+z^{2}-2 x y-2 y z-$ $2 z x$ is the magnitude of the 3 -momentum of the incoming particle in the c.m. frame. In the c.m. frame, using the energy momentum-conserving delta function and integrating over the final momenta, we have

$$
\begin{aligned}
W_{a b}(s)= & \frac{4 \sqrt{s} p_{a b}(s)}{1+\delta_{a b}} \int_{t_{\min }}^{t_{\max }} d t\left(\frac{d \sigma}{d t}\right) \\
& \times\left(1+f_{c}\left(E_{c}\right)\right)\left(1+f_{d}\left(E_{d}\right)\right),
\end{aligned}
$$

where

$$
\begin{aligned}
t_{\max , \min }= & m_{a}^{2}+m_{c}^{2}-\frac{1}{2 s}\left(s+m_{a}^{2}-m_{b}^{2}\right)\left(s+m_{c}^{2}-m_{d}^{2}\right) \\
& \pm \frac{1}{2 s} \sqrt{\lambda\left(s, m_{a}^{2}, m_{b}^{2}\right) \lambda\left(s, m_{c}^{2}, m_{d}^{2}\right)}
\end{aligned}
$$

In the limit of constant $|M|^{2}$, Eq. (44) reduces to

$$
\begin{aligned}
W_{a b}(s)= & \frac{1}{1+\delta_{a b}} \frac{|M|^{2}}{16 \pi \sqrt{s} p_{a b}}\left(t_{\max }-t_{\min }\right)\left(1+f_{c}\left(E_{c}\right)\right) \\
& \times\left(1+f_{d}\left(E_{d}\right)\right),
\end{aligned}
$$

and the transition frequency or the inverse relaxation time is given as

$$
\begin{aligned}
\omega\left(E_{a}\right) \equiv & \tau\left(E_{a}\right)^{-1}=\frac{1}{256 \pi^{3} E_{a}} \int_{m_{b}}^{\infty} d E_{b} \sqrt{E_{b}^{2}-m_{b}^{2}} f\left(E_{b}\right)|M|^{2} \\
& \times \int_{-1}^{1} \frac{d x}{1+\delta_{a b}} \frac{1}{p_{a b} \sqrt{s}}\left(t_{\max }-t_{\min }\right) .
\end{aligned}
$$

In the above,

$$
s=2 E_{a} E_{b}\left(1+\frac{m_{a}^{2}+m_{b}^{2}}{2 E_{a} E_{b}}-\frac{p_{a} p_{b}}{E_{a} E_{b}} x\right) .
$$

To calculate, e.g., the $\pi^{+}$relaxation time $\left(\tau_{\pi^{+}}\right)$, we consider the scattering processes $\pi^{+}+\pi^{i} \rightarrow \pi^{+}+\pi^{i}(i=+,-, 0)$ and $\pi^{+}+\sigma \rightarrow \pi^{+}+\sigma$.

To get an order of magnitude of the average relaxation time, one can also calculate an energy-averaged mean interaction frequency for a given species as $\bar{\omega}_{a} \equiv \bar{\tau}_{a}{ }^{-1}$ as

$$
\bar{\omega}_{a}=\frac{1}{n_{a}} \int \frac{d \mathbf{p}}{(2 \pi)^{3}} \omega_{a}\left(E_{a}\right) f_{a}\left(E_{a}\right),
$$

with

$$
n_{a}=\int \frac{d \mathbf{p}}{(2 \pi)^{3}} f_{a}\left(E_{a}\right)
$$




\section{B. Relaxation time estimation-Quark scatterings}

We next consider the quark scattering within the model through the exchange of pion and sigma meson resonances. The approach is similar to Refs. [25,35,59] performed within the NJL model to estimate the corresponding relaxation time for the quarks and antiquarks. The transition frequency is again given by Eq. (41), with the corresponding $W_{a b}$ given as

$$
\begin{aligned}
W_{a b}^{q}(s)= & \frac{2 \sqrt{s\left(s-4 m^{2}\right)}}{1+\delta_{a b}} \int_{t_{\min }}^{0} d t\left(\frac{d \sigma}{d t}\right)\left(1-f_{c}\left(\frac{\sqrt{s}}{2}, \mu\right)\right) \\
& \times\left(1-f_{d}\left(\frac{\sqrt{s}}{2}, \mu\right)\right)
\end{aligned}
$$

where

$$
\frac{d \sigma}{d t}=\frac{1}{16 \pi s\left(s-4 m^{2}\right)} \frac{1}{p_{a b}^{2}}|\bar{M}|^{2},
$$

with the corresponding suppression factors appropriate for fermions. For the quark scatterings, in the present case for two flavors, we consider the following scattering processes:

$$
\begin{aligned}
& u \bar{u} \rightarrow u \bar{u}, \quad u \bar{d} \rightarrow u \bar{d}, \quad u \bar{u} \rightarrow d \bar{d}, \\
& u u \rightarrow u u, \quad u d \rightarrow u d, \quad \bar{u} \bar{u} \rightarrow \bar{u} \bar{u}, \\
& \bar{u} \bar{d} \rightarrow \bar{u} \bar{d}, \quad d \bar{d} \rightarrow d \bar{d}, \quad d \bar{d} \rightarrow u \bar{u}, \\
& d \bar{u} \rightarrow d \bar{u}, \quad d d \rightarrow d d, \quad \bar{d} \bar{d} \rightarrow \bar{d} \bar{d} .
\end{aligned}
$$

One can use $i$-spin symmetry, charge conjugation symmetry, and crossing symmetry to relate the matrix element square for the above 12 processes to get them related to one another, and one has to evaluate only two independent matrix elements to evaluate all the 12 processes. We choose these, as in Ref. [59], to be the processes $u \bar{u} \rightarrow u \bar{u}$ and $u \bar{d} \rightarrow u \bar{d}$ and use the symmetry conditions to calculate the rest. We note, however, that, while the matrix elements are related, the thermal-averaged rates are not, as they involve also the thermal distribution functions for the initial states as well as the Pauli blocking factors for the final states. We also write down the square of the matrix elements for these two processes explicitly $[25,59]$ :

$$
\begin{aligned}
\left|\bar{M}_{u \bar{u} \rightarrow u \bar{u}}\right|^{2}= & g_{\sigma}^{4}\left[s^{2}\left|D_{\pi}(\sqrt{s}, 0)\right|^{2}+t^{2}\left|D_{\pi}(0, \sqrt{-t})\right|^{2}\left(s-4 m^{2}\right)^{2}\left|D_{\sigma}(\sqrt{s}, 0)\right|^{2}+\left(t-4 m^{2}\right)^{2}\left|D_{\sigma}(0, \sqrt{-t})\right|^{2}\right. \\
& +\frac{1}{N_{c}} \operatorname{Re}\left(s t D_{\pi}^{*}(\sqrt{s}, 0) D_{\pi}(0, \sqrt{-t})+s\left(4 m^{2}-t\right) D_{\pi}^{*}(\sqrt{s}, 0) D_{\sigma}(0, \sqrt{-t})\right. \\
& \left.\left.+t\left(4 m^{2}-s\right) D_{\pi}(0, \sqrt{-t}) D_{\sigma}^{*}(\sqrt{s}, 0)+\left(4 m^{2}-s\right)\left(4 m^{2}-t\right) D_{\sigma}(0, \sqrt{-t}) D_{\sigma}^{*}(\sqrt{s}, 0)\right)\right] .
\end{aligned}
$$

Similarly, the same for the process $u \bar{d} \rightarrow u \bar{d}$ is given as [59]

$$
\begin{aligned}
\left|\bar{M}_{u \bar{d} \rightarrow u \bar{d}}\right|^{2}= & g_{\sigma}^{4}\left[4 s^{2}\left|D_{\pi}(\sqrt{s}, 0)\right|^{2}+t^{2}\left|D_{\pi}(0, \sqrt{-t})\right|^{2}\left(s-4 m^{2}\right)^{2}\left|D_{\sigma}(\sqrt{s}, 0)\right|^{2}+\left(t-4 m^{2}\right)^{2}\left|D_{\sigma}(0, \sqrt{-t})\right|^{2}\right. \\
& \left.+\frac{1}{N_{c}} \operatorname{Re}\left(-2 s t D_{\pi}^{*}(\sqrt{s}, 0) D_{\pi}(0, \sqrt{-t})+2 s\left(4 m^{2}-t\right) D_{\pi}^{*}(\sqrt{s}, 0) D_{\sigma}(0, \sqrt{-t})\right)\right] .
\end{aligned}
$$

The meson propagators $D_{a}(\sqrt{s}, 0),(a=\sigma, \pi)$ are given by

$$
D_{a}(\sqrt{s}, \mathbf{0})=\frac{i}{s-M_{a}^{2}-i \operatorname{Im} \Pi_{M_{a}}(\sqrt{s}, \mathbf{0})} .
$$

In the above, the masses of the mesons are given by Eqs. (23) and (24) determined by the curvature of the thermodynamic potential. Further, in Eq. (53), $\operatorname{Im} \Pi(\sqrt{s}, 0)$, which is related to the width of the resonance as $\Gamma_{a}=\operatorname{Im} \Pi_{a} / M_{a}$, is given as [59]

$$
\begin{aligned}
\operatorname{Im} \Pi_{a}(\omega, \mathbf{0})= & \theta\left(\omega^{2}-4 m^{2}\right) \frac{N_{c} N_{f}}{8 \pi \omega}\left(\omega^{2}-\epsilon_{a}^{2}\right) \sqrt{\omega^{2}-4 m^{2}} \\
& \times\left(1-f_{-}(\omega)-f_{+}(\omega)\right),
\end{aligned}
$$

with $\epsilon_{a}=0$ for pions and $\epsilon_{a}=2 m$ for sigma mesons.

With the squared matrix elements for the quark scatterings given as above, the transition frequency for the quark of a given species is

$$
\omega_{q}\left(E_{a}\right)=\frac{1}{2 E_{a}} \int d \pi_{b} f\left(E_{b}\right) W_{a b}^{q} .
$$




\section{Quark pion scattering and relaxation time}

Next, we compute the contribution of quark meson scattering to the relaxation times for both mesons as well as quarks. One can argue that the dominant contribution comes from pions as their number is large compared to the sigma mesons both below and above $T_{c}$. Therefore, in the following, we consider the quark pion scattering only. The Lorentz-invariant scattering matrix element can be written as $\bar{U}\left(p_{2}\right) T_{b a} U\left(p_{1}\right)$, with $\bar{U} U=2 m_{q}$ and with $p_{1}, p_{2}$ denoting the initial and final the quark momenta, respectively, and $q_{1}$ and $q_{2}$, being the momenta of the pions,

$T_{b a}=\delta_{b a} \frac{1}{2}\left(q_{1}+q_{2}\right)^{\mu} \gamma_{\mu}\left(\delta_{a b} B^{(+)}+i \epsilon_{a b c} \tau_{c} B^{(-)}\right)$,

where

$$
B^{(+)}=g_{\sigma}^{2}\left(\frac{1}{u-m_{q}^{2}}-\frac{1}{s-m_{q}^{2}}\right),
$$

and

$$
B^{(-)}=-g_{\sigma}^{2}\left(\frac{1}{u-m_{q}^{2}}+\frac{1}{s-m_{q}^{2}}\right) .
$$

Averaging over the spin and isospin factors, the matrix element square for the quark pion scattering is given by

$$
|\bar{M}|^{2}=\frac{g_{\sigma}^{4}}{6}\left((s-u)^{2}-t\left(t-4 m_{\pi}^{2}\right)\right)\left(3 B_{+}^{2}+2 B_{-}^{2}\right) .
$$

The corresponding transition frequency is given by

$$
\omega_{q \pi}\left(E_{a}\right)=\frac{1}{2 E_{a}} \int d \pi_{b} f\left(E_{b}\right) W_{a b}^{(q-\pi)},
$$

where

$W_{a b}^{(q-\pi)}=\frac{1}{8 \pi} \times \frac{1}{2 \sqrt{s} p_{0}} \int d t\left|\bar{M}_{q-\pi}\right|^{2}\left(1-f_{q}\right)\left(1+f_{\pi}\right)$.

In the above, $p_{0}^{2}=\left(s+m_{q}^{2}-m_{\pi}^{2}\right)^{2} /(4 s)-m_{q}^{2}$. The scattering will contribute to both the quark relaxation time as well as to the pion relaxation time using Eq. (60) with appropriate modification for the initial state.

Let us note that there are poles in the $\mathrm{u}$ channel in the quark pion scattering term beyond the critical temperature when the pion mass becomes larger than the quark mass. However, this is taken care of once we include the imaginary part of the quark self-energy in the propagators for the quarks in the calculation of the amplitude in Eqs. (57) and (58). The quark self-energy due to scattering with mesons can be written as [30]

$$
\Sigma\left(p_{0}, \mathbf{p}\right)=m \Sigma_{0}+\gamma \cdot \mathbf{p} \Sigma_{3}-\gamma_{0} p_{0} \Sigma_{4}
$$

so that the quark propagators get modified as

$$
\begin{aligned}
S\left(p_{0}, \mathbf{p}\right) & =\frac{1}{\not p-m-\Sigma} \\
& =\frac{m\left(1+\Sigma_{0}\right)+\gamma_{0} p_{0}\left(1+\Sigma_{4}\right)-\gamma \cdot \mathbf{p}\left(1+\Sigma_{3}\right)}{p_{0}^{2}\left(1+\Sigma_{4}\right)^{2}-\mathbf{p}^{2}\left(1+\Sigma_{3}\right)^{2}-m^{2}\left(1+\Sigma_{0}\right)^{2}} .
\end{aligned}
$$

The imaginary part of the dimensionless $\Sigma_{j},(j=0,3,4)$, is given as

$$
\begin{aligned}
\operatorname{Im} \Sigma_{j}\left(p_{0}, \mathbf{p}\right) & =\frac{g^{2}}{32 \pi p} d_{j} \int_{E_{\min }}^{E_{\max }} d E_{f} C_{j}\left[f_{b}\left(E_{b}\right)\right. \\
& \left.+f_{-}\left(E_{f}\right)+f_{+}\left(E_{f}\right)\right] .
\end{aligned}
$$

In the above, $E_{b}=E_{f}+p_{0}, p_{0}=\sqrt{\mathbf{p}^{2}+m^{2}}$ and $f_{ \pm}$are the distribution functions for the quarks/antiquarks, $f_{b}$ is the meson distribution function, and, $C_{j}$ 's are weight factors given as

$C_{0}=1, \quad C_{3}=\frac{m_{M}^{2}-2 m^{2}-2 E_{f} p_{0}}{2 \mathbf{p}^{2}}, \quad C_{4}=-\frac{E_{f}}{p_{0}}$.

The integration limits are given by

$E_{\max , \min }=\frac{1}{2 m^{2}}\left[\left(m_{M}^{2}-2 m^{2}\right) p_{0} \pm|\mathbf{p}| m_{M} \sqrt{m_{M}^{2}-4 m^{2}}\right]$.

Further, the degeneracy factors $d_{3,4}$ are 3 for pions and 1 for sigma, while $d_{0}$ is -3 for pions and 1 for the sigma meson. To calculate the total relaxation time for a quark of species a, we compute the total interaction frequency as $\omega_{q}^{\text {total }}\left(E_{a}\right)=\omega\left(E_{a}\right)+\omega_{q \pi}\left(E_{a}\right)$. One can define an average relaxation time for the quarks similar to Eq. (47) as $\bar{\tau}_{q}^{\text {total }}=\frac{1}{\bar{\omega}_{q}^{\text {total }}}$,

$$
\bar{\omega}_{q}^{\text {total }}=\frac{1}{n_{q}} \int \frac{d \mathbf{p}}{(2 \pi)^{3}} f_{q}(E) \omega_{q}^{\text {total }}(E) .
$$

\section{RESULTS}

\section{A. Meson scatterings}

Let us first discuss the results arising from meson scattering alone. Using Eqs. (46), with constant $|M|^{2}$ as discussed, we have plotted the average relaxation times for the $\sigma$ meson and $\boldsymbol{\pi}$ mesons in Fig. 5. The relaxation times are minimum at the transition temperature. Because of larger mass of $\sigma$ mesons below the transition temperature, $\bar{\tau}_{\sigma}$ is much larger as compared to $\bar{\tau}_{\boldsymbol{\pi}}$. They become almost degenerate after the chiral transition, as may be expected from the behavior of their masses beyond the transition 


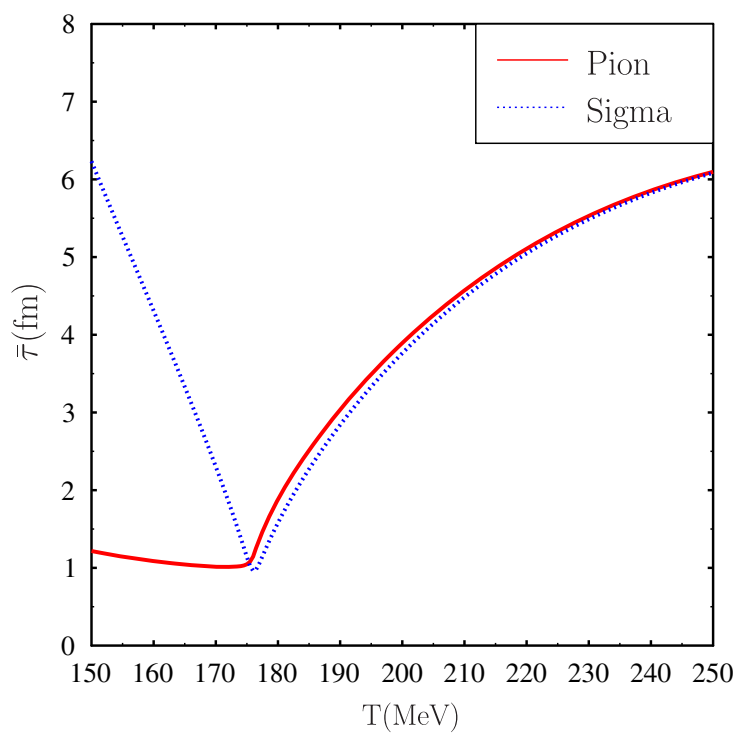

FIG. 5. Average relaxation time for pions (solid line) and sigma meson (dotted line). Only meson-meson scatterings are considered here.

temperature. We may comment here that the particle with larger relaxation time dominates the viscosities as it can transport energy and momentum to larger distances before interacting. In Fig. 6, we have shown the behavior of the specific viscosities (normalized to entropy density) as a function of temperature. In Fig. 6(a), we have plotted the temperature dependence of the ratio $\eta / s$ for $\mu=0$. The behavior of this ratio is essentially determined by the behavior of the relaxation time. Similar to Fig. $5, \eta / s$ shows

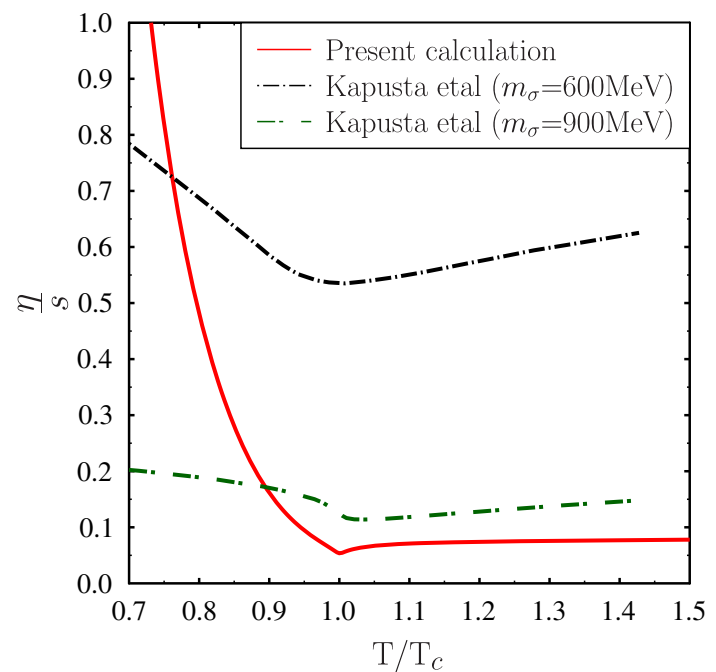

(a) a minimum at the crossover temperature, and the value at the minimum is about $\eta / s \sim 0.053$, which is slightly lower than the KSS bound of $1 / 4 \pi$. We note that we have considered here only the contributions from meson scatterings. As we shall see later, inclusion of quark degrees of freedom increases the ratio. We have also compared with linear sigma model calculations [38] in which the quark as well as Polyakov loop contributions are not taken into account. The general behavior of the present calculations is similar to earlier calculations in the sense of having a minimum at the chiral crossover temperature. However, the magnitude of the ratio at the critical temperature is smaller compared to Ref. [38]. This is probably due to the fact that the entropy density in the present calculations has contributions including those of the gluon included through the Polyakov loop potential. The large entropy density, we believe, decreases the magnitude of the ratio.

In Fig. 6(b), the ratio of bulk viscosity to entropy is plotted, which shows a maximum at the transition temperature. We have also plotted in the same figure the results without quarks and the Polyakov loop potential. The present results show a distinct peak structure in the $\zeta / s$ ratio at the crossover temperature. Let us note that such a peak is expected as an effect of large conformality violation at the transition temperature as indicated in lattice simulations [28,60]. In Ref. [38], a peak structure is seen for a heavier sigma meson $\left(m_{\sigma}=900 \mathrm{MeV}\right)$, which was interpreted as an effect of stronger self-coupling $\lambda$ for higher $M_{\sigma}$. However, in the present case, this arises with quark and Polyakov loop degrees of freedom even with a lighter $M_{\sigma}=600 \mathrm{MeV}$. The other characteristic feature of the

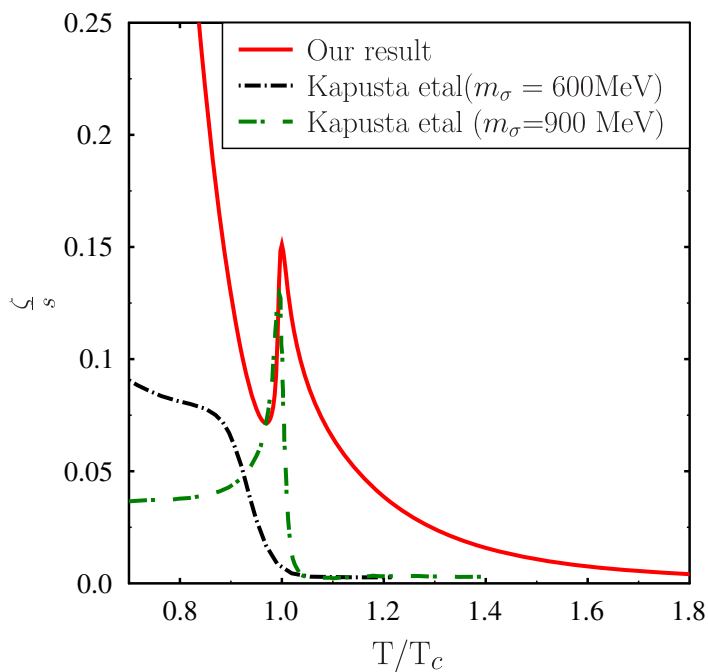

(b)

FIG. 6. Computations show mesonic contribution calculated using only meson-meson interactions. (a) Shear viscosity-to-entropy ratio for $\mu=0$. Present results are shown by solid lines. The two dot dashed curves correspond to results of the linear sigma model of Ref. [38] corresponding two different masses for sigma mesons. (b) Bulk viscosity-to-entropy ratio for $\mu=0$. Results for current calculations are shown by the solid line. The other results correspond to the work by Kapusta et al. (short dashed line) of the linear sigma model with $\left(m_{\sigma}=600 \mathrm{MeV}\right)$ and Kapusta et al. (dash dot curve) for the linear sigma model with $m_{\sigma}=900 \mathrm{MeV}$ [38]. 
present calculation is that, beyond the critical temperature, the ratio $\zeta / s$ falls at a slower rate as compared to results of previous calculations. This has to do with the fact that the velocity of sound approaches the ideal gas limit slowly as the effect of Polyakov loops on the quark distribution function remains significant beyond the critical temperature. In fact, at the transition temperature, the value of the Polyakov loop remains about half its value of the ideal limit. Apart from this, the masses of mesons also get affected by the quark distribution functions significantly beyond the critical temperature. These nonideal effects lead to a slower decrease of the ratio beyond the critical temperature.

\section{B. Quark scatterings}

Next, we discuss quark scattering. In Fig. 7, we show the behavior of average relaxation time for quark scattering. The quark scattering through the exchange of mesons is shown by the solid line in the figure. Let us recall that the average relaxation time is inversely proportional to the transition rate, which is related to the cross section. The dominant contribution here comes from the quarkantiquark scattering from the $s$ channels through propagation of the resonance states, the pions, and the sigma mesons. The masses of the sigma meson decrease with temperature, becoming a minimum at the transition temperature, leading to an enhancement of the cross section. Beyond this, the cross section decreases due to the increase in the masses of the mesons. This, in turn, leads to a minimum in the relaxation time.

The average relaxation time for quarks including the quark meson scattering along with the quark scattering is shown as the dashed curve in Fig. 7. This curve lies below the quark-quark scattering curve as there is an additional contribution to the transition rate from the quark meson scattering. Below the critical temperature, the quark meson scattering dominates over the quark-quark scattering due to the smaller mass of the pions as compared to the massive constituent quarks. Beyond the critical temperature, one would have expected the quark meson scattering contribution to be negligible because of the suppression due to the large meson masses. However, as was noted earlier, beyond the critical temperature, there are poles in the scattering amplitude in the $u$ channel for quark-pion scattering as the pion mass becomes larger than the quark masses. This is, however, regulated by the finite width of the quarks as calculated in Eq. (62). Nonetheless, the contribution of the quark-pion scattering to the total quark interaction frequency $\omega_{q \pi}(\mathrm{E})$ is non-negligible beyond the critical temperature.

We next discuss the contribution of different scatterings to the specific shear viscosity $\eta / s$. The same is shown in Fig. 8(a) for vanishing chemical potential. The contribution from the mesons to the shear viscosity arising from the meson scatterings only is shown by the green dashed curve,

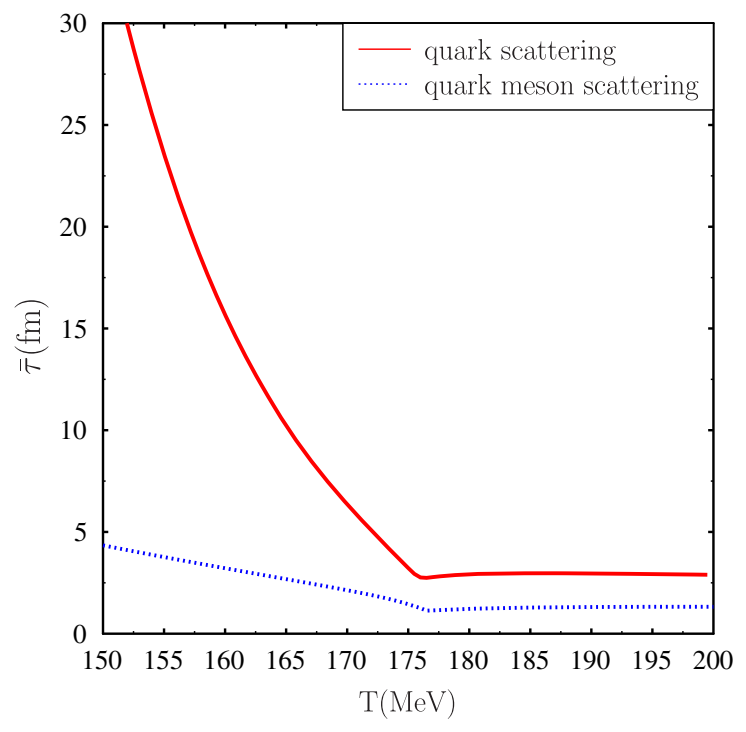

FIG. 7. Average relaxation time for quarks arising from quark scattering. The solid curve corresponds to quark quark-quark and quark-antiquark scattering with meson exchange. The dashed curve corresponds to including the effect of quark meson scatterings. Both the curves correspond to the $\mu=0$ case.

while the effect of including the meson-quark scattering is shown by the maroon dotted curve. Similarly, the quark contribution to this ratio $\eta / s$ arising from quark-quark scattering only is shown by the red solid line, while the total contributions including the quark-pion scattering is shown by the blue dotted line. This also demonstrates the importance of the scattering of quarks and mesons to the total viscosity coefficient. The total contributions from both the quarks and mesons is shown as the black dashed curve in Fig. 8.

In a similar manner, various contributions to the specific bulk viscosity $(\zeta / s)$ coefficient are shown in Fig. 8(b). As may be observed, while no peak structure is seen for this coefficient from the contributions arising from quarks scatterings only, such a structure is seen only when one includes the quark meson scattering. The total effect is shown as a black dashed curve in Fig. 8(b).

In Fig. 9, we compare the present results with earlier works on the NJL model. As may be noted, in general, the behavior is similar regarding the shear viscosity-toentropy ratio. Both NJL as well as the present calculations of the PQM model show the similar behavior of having a minimum at the transition temperature as in Refs. [24,25]. The results of Ref. [35], on the other hand, show a monotonic decrease with temperature. The bulk viscosityto-entropy ratio here, however, shows a much faster rise as the temperature is lowered below the critical temperature. In fact, both the specific viscosities rise much faster compared to NJL models below the critical temperature in the PQM model considered here. The reason could be due to the fact that the entropy density for the PQM model is smaller compared to NJL models. The Polyakov loop 


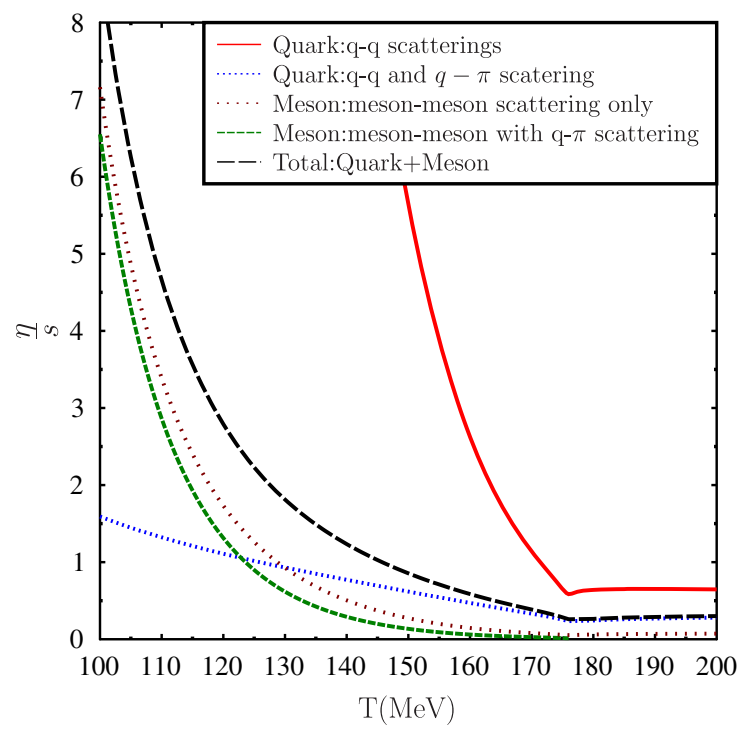

(a)

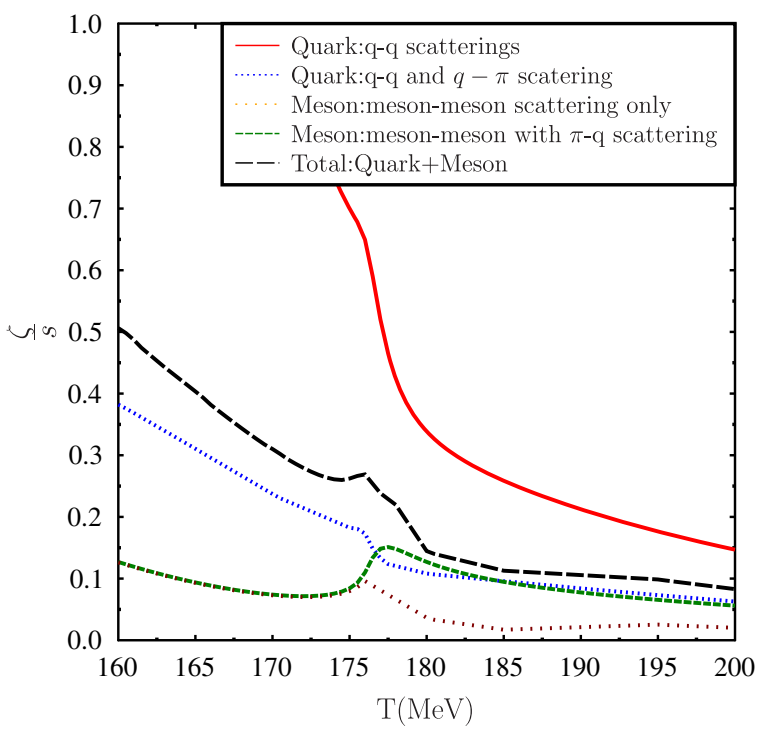

(b)

FIG. 8. Different contributions for specific viscosity coefficients. $\eta / s$ is shown in the left, while $\zeta / s$ is shown on the right. In both the figures, contributions from the quarks with relaxation time computed using only quark-quark scattering(red solid line) and also including quark-meson scattering(blue dotted line) are shown as a function of temperature. The contribution of the mesons due to meson-meson scattering (green dashed curve) and including meson-quark scattering (maroon short dashed curve) is also shown. The total contribution from the quarks and mesons is shown by the black long dashed curve. All the curves correspond to the $\mu=0$ case.

decreases as the temperature is lowered, which leads to a suppression of quark distribution functions, leading to decrease of entropy density at a faster rate as compared to the NJL model. Moreover, within the present approximation, pions do not contribute to the thermodynamics

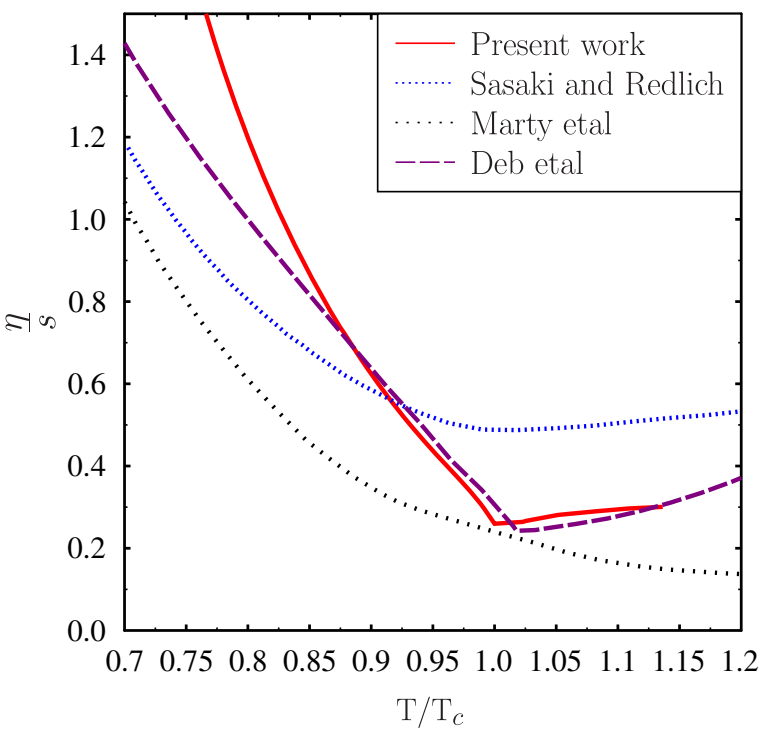

(a) here. Further, for temperature larger than the critical temperature, the bulk viscosity vanishes slowly with an increase in temperature as compared to the NJL model. This is due to the fact that the Polyakov loop variable takes its asymptotic values only at very high temperatures.

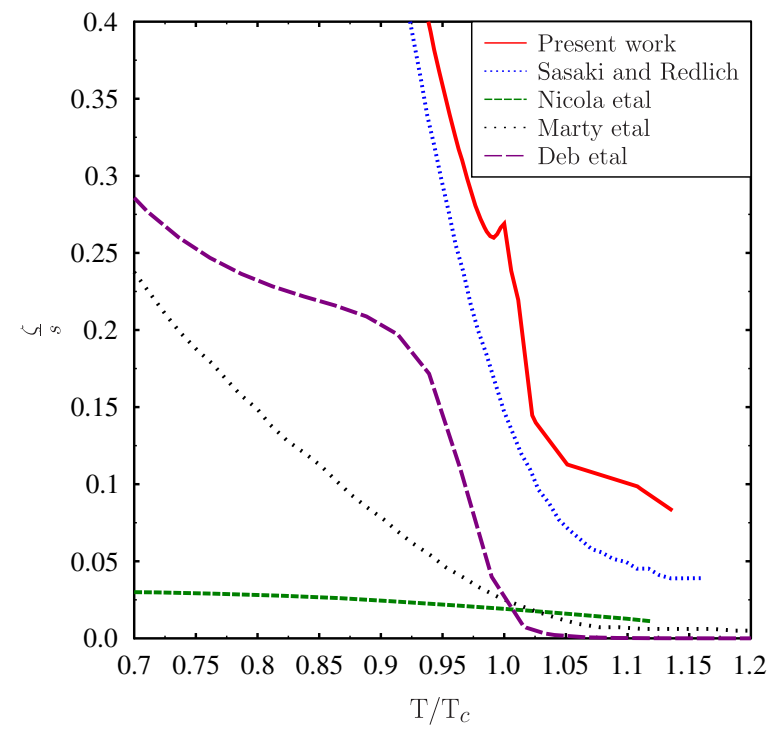

(b)

FIG. 9. (a) Shear viscosity-to-entropy ratio for $\mu=0$. Present results are shown by solid lines. The dotted line corresponds to results of the NJL model of Ref. [24], the short dashed curve corresponds to results of Marty et al. Ref. [35], and the long dashed curves correspond to the results of Deb et al. of Ref. [25]. (b) The results of the bulk viscosity-to-entropy ratio compared with other results in NJL models. The notation is similar to that of (a). 


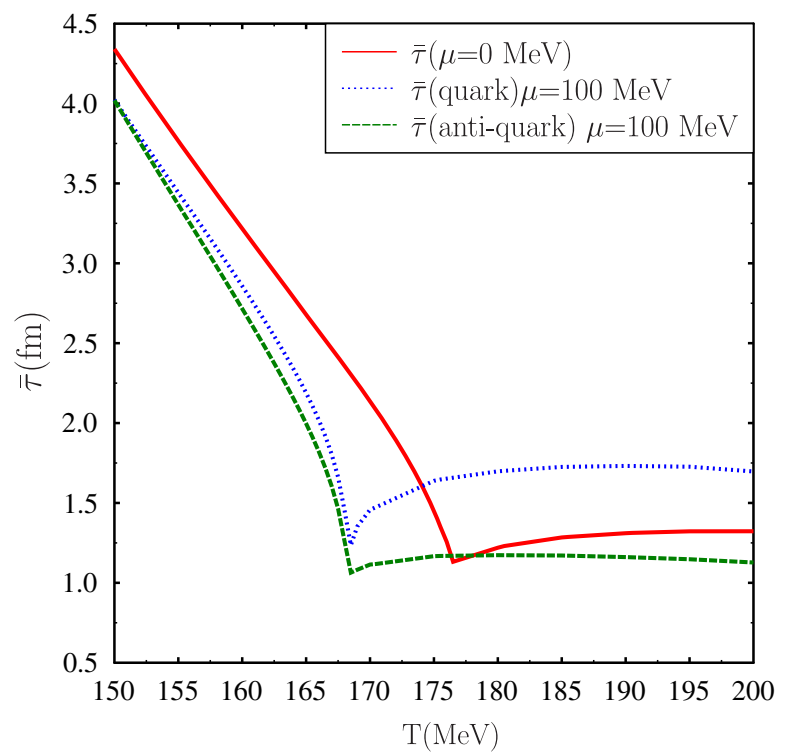

FIG. 10. Average relaxation time of quarks and antiquarks for $\mu=100 \mathrm{MeV}$. The solid line corresponds to the case of $\mu=0 \mathrm{MeV}$.

Next, we discuss the effect of finite chemical potential on the transport coefficients. To begin with, let us note that the average relaxation time $\bar{\tau}_{a}$ as in Eq. (67) depends both on the transition rate and the density of the particles in the initial state. To this end, let us discuss the case of $\mathrm{T}>\mathrm{T}_{c}$. Here, the quark densities are larger than those of antiquarks. Further, the dominant contribution in this range of temperatures arises from $u \bar{d} \rightarrow u \bar{d}$ scatterings. As there are fewer antiquarks to scatter off, the average transition frequency of quark-antiquark scattering decreases. This

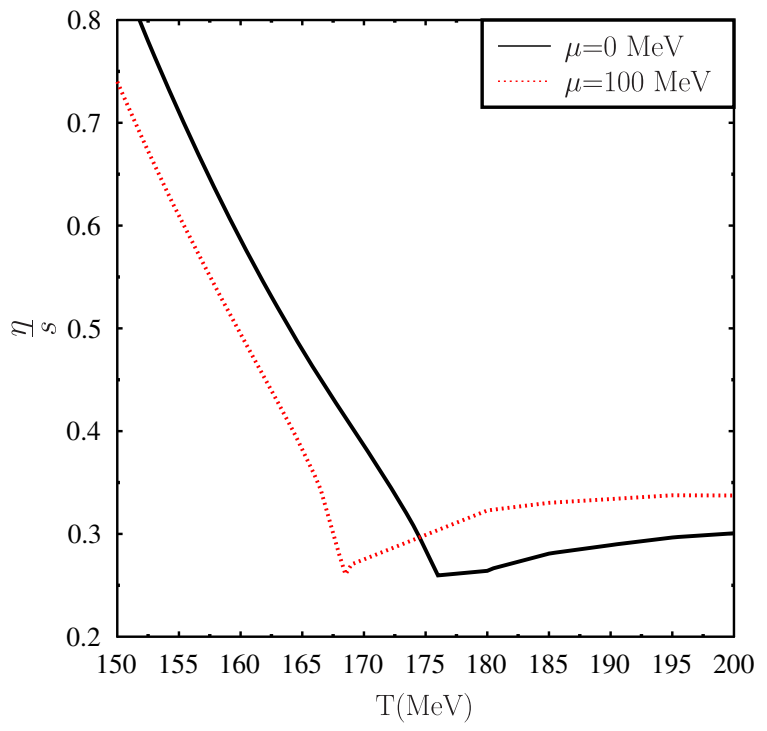

(a) leads to $\bar{\tau}_{q}(\mu)>\bar{\tau}_{q}(\mu=0)$. On the other hand, for the antiquarks, there are more quarks to scatter off than compared to the case of $\mu=0$. Hence, this leads to $\bar{\tau}_{\bar{q}}(\mu)<\bar{\tau}_{\bar{q}}(\mu=0)$. This expected behavior is seen in Fig. 10. Next, let us consider the case $\mathrm{T}<\mathrm{T}_{c}$. In this case, the antiquark density is heavily suppressed due to constituent quark mass, and the chemical potential and dominant contribution for quark relaxation time therefore arises from quark-quark scatterings. This leads to $\bar{\tau}_{q}(\mu)<\bar{\tau}_{q}(\mu=0)$. On the other hand, for the antiquarks, though their number density is smaller, their interaction frequency is enhanced both by the larger amplitude for $\mathbf{M}_{u \bar{d} \rightarrow u \bar{d}}$ scattering and the larger number of quarks as compared to case at $\mu=0$. This leads to $\bar{\tau}_{\bar{q}}(\mu)<$ $\bar{\tau}_{q}(\mu=0)<\bar{\tau}_{\bar{q}}(\mu=0)$. This general behavior is reflected in the average relaxation time dependence on $\mathrm{T}$ in Fig. 10 below the critical temperature.

In Fig. 11, we have shown the results for the viscosities at $\mu=100 \mathrm{MeV}$. Figure 11(a) shows the variation of the specific shear viscosity $(\eta / s)$ as a function of temperature for zero and finite chemical potential. The behavior of shear viscosity essentially follows that of the behavior of the relaxation time. $\eta / s$ has a minimum at the critical temperature with $\eta /\left.s\right|_{\min } \sim 0.23(\mu=0)$ due to suppression of the scattering cross section at higher temperature. At finite $\mu$, the ratio is a little higher as compared to the value at vanishing $\mu$. This is due to two reasons. First, the relaxation time at nonzero chemical potential is larger, and, moreover, the quark density also becomes larger at finite chemical potential. At temperatures below the critical temperature and near the critical temperature, $\eta / s(\mu)<\eta / s(\mu=0)$ as the relaxation time is lower. However, at lower

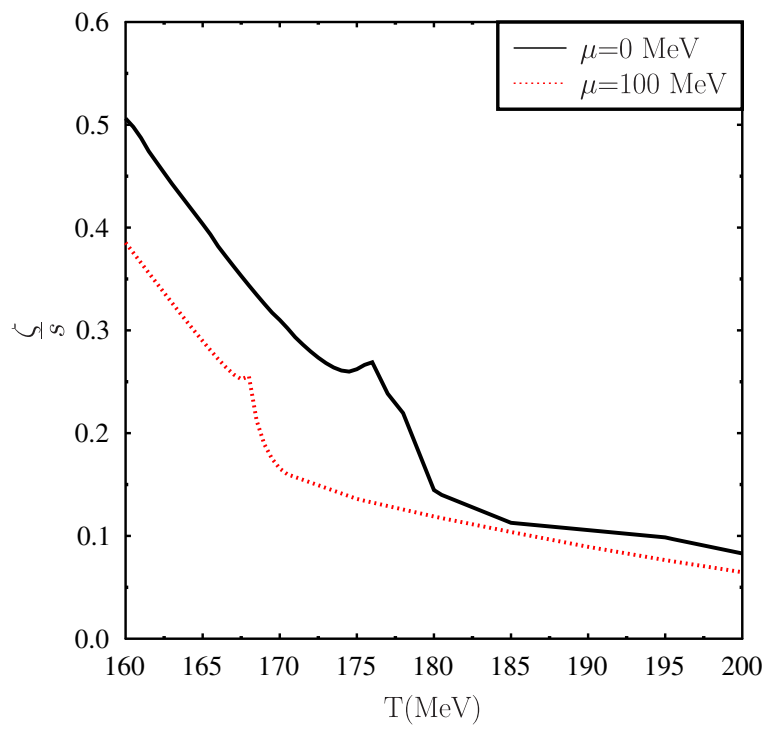

(b)

FIG. 11. Viscosities for $\mu=100 \mathrm{MeV}$. The left figure shows $\eta / s$ as a function of temperature for $\mu=0 \mathrm{MeV}$ (solid line) and $\mu=100 \mathrm{MeV}$ (dotted line). The right figure shows the ratio $\zeta / s$ as a function of temperature. 
temperatures, the meson scattering becomes significant, and $\eta / s$ for finite chemical potential becomes similar to that at vanishing chemical potential as is observed in the figure.

In Fig. 11(b), we have plotted the bulk viscosity-toentropy ratio for $\mu=0 \mathrm{MeV}$ and $\mu=100 \mathrm{MeV}$. It turns out that at finite $\mu$ the specific bulk viscosity is smaller than the value at $\mu=0 \mathrm{MeV}$. The reason for it is the fact that the dominating contribution to the finite $\mu$ arises from the term $M^{2}-T M \frac{d M}{d T}-\mu M \frac{d M}{d \mu}$ in the expression for $\zeta / s$ in Eq. (35). This is due to the sharp variations of the order parameters at finite chemical potential as may be observed in Fig. 3. As this term contributes negatively to the expression for $\zeta$, the specific bulk viscosity at finite $\mu$ is lower than that at $\mu=0 \mathrm{MeV}$.

In Fig. 12, we have shown the results for thermal conductivity. We have plotted here the dimensionless quantity $\lambda / T^{2}$ as a function of temperature. We have plotted the results for $\mu=100 \mathrm{MeV}$. As is well known, thermal conduction, which involves the relative flow of energy and baryon number, vanishes at zero baryon density. In fact, $\lambda$ diverges as $1 / n^{2}$, as may be expected from the expression given in Eq. (36). However, in the dissipative current, the conductivity occurs as $\lambda n^{2}$ [61,62], and the heat conduction vanishes for $\mu=0$ [63]. On the other hand, in some cases, such as when the pion number is conserved, heat conduction can be sustained by pions. In the presence of a pionic chemical potential corresponding to a conserved pion number, thermal conductivity can be nonzero at vanishing baryonic chemical potential. This has been the basis for the estimation of thermal conductivity at zero baryon density but finite pion density $[31,40,47]$. However, in the present case, we consider the case of vanishing pion chemical potential and show only the contribution of quarks to thermal conductivity.

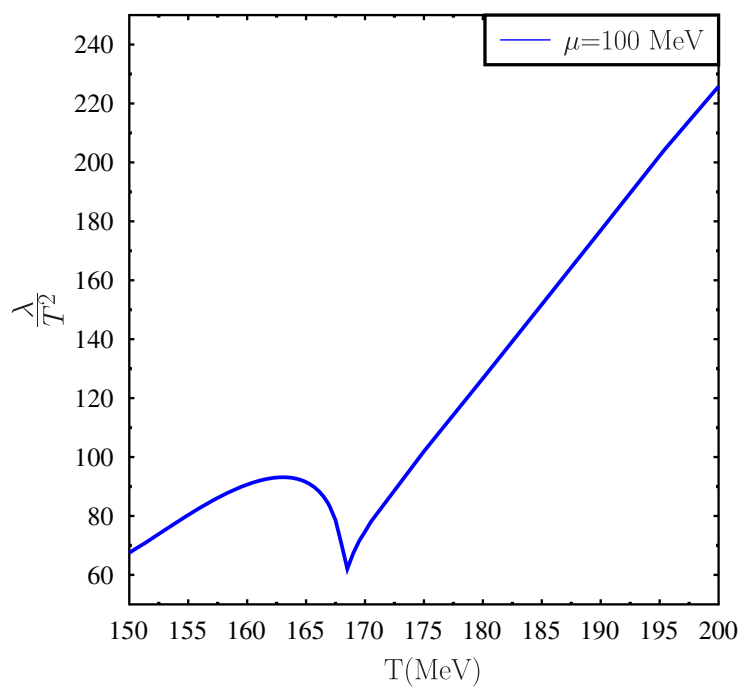

FIG. 12. Thermal conductivity in units of $T^{2}$ as a function of temperature for $\mu=100 \mathrm{MeV}$.
As expected from the behavior of the relaxation time, the specific thermal conductivity has a minimum at the critical temperature similar to Ref. [25] for the NJL model. The sharp rise of $\lambda / \mathrm{T}^{2}$ can be understood by performing a dimensional argument to show that at very high temperature when chiral symmetry is restored the integral increases as $\mathrm{T}^{3}$ while the prefactor $w /(n T)$ grows as $\mathrm{T}^{2}$ for small chemical potentials. Apart from this kinematic consideration, the integrand further is multiplied by $\tau(E)$, which itself is an increasing function of temperature beyond $T_{c}$. This leads to the sharp rise of the ratio $\lambda / T^{2}$ beyond the critical temperature. Below the critical temperature, however, the ratio decreases, which is in contrast to the NJL results of Ref. [25]. The reason is twofold. First, the magnitude of the relaxation time decreases when quark meson scattering is included as compared to quark-quark scattering as shown in Fig. 7. Apart from this, in the integrand, the distribution functions are suppressed by Polyakov loops as compared to the NJL model. As the antiquark densities are suppressed compared to quark densities at finite chemical potential, the high-temperature behavior is decided by the quark-quark scattering.

\section{SUMMARY}

Transport coefficients of hot and dense matter are important inputs for the hydrodynamic evolution of the plasma that is produced following a heavy ion collision. In the present study, we have investigated these coefficients, taking into account the nonperturbative effects related to chiral symmetry breaking as well as confinement properties of strong interaction physics within an effective model, the Polyakov loop extended quark meson coupling model. These coefficients are estimated using the relaxation time approximation for the solutions of the Boltzman kinetic equation.

We first calculated the medium-dependent masses of the mesons and quarks within a mean field approximation. The contribution of the mesons to the transport coefficients has been calculated through estimating the relaxation time for the mesons arising both from meson-meson scattering and meson-quark scattering. The contribution to the transport coefficients arises mostly from the meson scatterings at temperatures below the critical temperature, while above the critical temperature, the contributions arising from the quark scatterings become dominant. In particular, quark meson scattering contributes significantly to the relaxation time for the quarks both below and above the critical temperature. The quark-pion scattering above the critical temperature gives significant contribution due to the pole structure of the corresponding scattering amplitude.

One important approximation in the present analysis is that the kinetic terms for the mesons are not modified at finite temperature and meson dispersion relation remains similar to those at the zero-temperature relativistic dispersion relation. The only temperature effect that remains 
in the meson dispersion lies in the temperature-dependent meson masses obtained through the curvature of the effective potential [54]. A more realistic approach would be to use effective field theory to have different dispersion relations for the mesons [64] depending upon their velocities and calculate the scattering processes to estimate the viscosities. However, such an approach is beyond the scope of present work in which we have restricted ourselves to thermal and density effects included in the masses and widths for the mesons.

In general, the effect of Polyakov loops lies in suppressing the quark contribution below the critical temperature. This leads to, in particular, the suppression of thermal conductivity at lower temperature arising from quark scattering. The effect of Polyakov loop also is significant near and above the critical temperature. Indeed, both the quark masses as well as Polyakov loop order parameter remain significantly different from their asymptotic values near the critical temperature. It will be interesting to examine the consequences of such nonperturbative features on the transport coefficients of heavy quarks as well as on the collective modes of QGP above and near the critical temperature. Some of these works are in progress and will be reported elsewhere.

\section{ACKNOWLEDGMENTS}

The authors would like to acknowledge many discussions with Guru Prasad Kadam and Pracheta Singha. S. G. is financially supported by University Grants Commission Dr. D. S. Kothari Post Doctoral Fellowship (India), under Grant No. F4-2/2006 (BSR)/PH/15-16/0060.
[1] U. Heinz and R. Snellings, Annu. Rev. Nucl. Part. Sci. 63, 123 (2013).

[2] M. Gyulassy and L. McLerran, Nucl. Phys. A750, 30 (2005).

[3] H. Niemi, G. S. Denicol, P. Huovienen, E. Molnar, and D. H. Rischke, Phys. Rev. Lett. 106, 212302 (2011).

[4] L. P. Csernai, J. I. Kapusta, and L. D. McLerran, Phys. Rev. Lett. 97, 152303 (2006).

[5] M. Luzum and P. Romatschke, Phys. Rev. Lett. 103, 262302 (2009).

[6] P. Romatschke and U. Romatschke, Phys. Rev. Lett. 99, 172301 (2007); T. Hirano and M. Gyulassy, Nucl. Phys. A769, 71 (2006).

[7] C. Gale, S. Jeon, and B. Schenke, Int. J. Mod. Phys. A 28, 1340011 (2013).

[8] B. Friman et al., The CBM Physics Book: Compressed Baryonic Matter in Laboratory Experiments, Lecture Notes in Physics (Springer, Berlin, 2011).

[9] D. Blaschke, J. Aichelin, E. Bratkovskaya, V. Friese, M. Gazdzicki, J. Randrup, O. Rogachevsky, O. Teryaev, and V. Toneev, Eur. Phys. J. A 52, 267 (2016).

[10] S. Sarkar and R. Sharma, Phys. Rev. D 96, 094025 (2017).

[11] H. Heiselberg and C. Pethick, Phys. Rev. D 48, 2916 (1993).

[12] N. Chamel and P. Hansel, Living Rev. Relativity 11, 10 (2008).

[13] D. Page and S. Reddy, Annu. Rev. Nucl. Part. Sci. 56, 327 (2006).

[14] D. G. Yakovlev, A. D. Kaminker, O. Y. Gnedin, and P. Haensel, Phys. Rep. 354, 1 (2001).

[15] D. G. Yakovlev, O. Y. Gnedin, A. D. Kaminker, K. P. Levenfish, and A. Y. Potekhin, Adv. Space Res. 33, 523 (2004).

[16] N. Andersson, Astrophys. J. 502, 708 (1998); N. Andersson and K. D. Kokkotas, Mon. Not. R. Astron. Soc. 299, 1059 (1998).

[17] T. K. Jha, H. Mishra, and V. Sreekanth, Phys. Rev. C 82, 025803 (2010).
[18] J. I. Kapusta, Relativistic Nuclear Collisions, LandoltBornstein New Series, edited by R. Stock (Springer-Verlag, Berlin, 2010), Vol. I/23.

[19] R. Kubo, J. Phys. Soc. Jpn. 12, 570 (1957).

[20] P. Kovtun, D. T. Son, and A. O. Starinets, Phys. Rev. Lett. 94, 111601 (2005).

[21] P. Arnold, G. D. Moore, and L. G. Yaffe, J. High Energy Phys. 11 (2000) 001; 01 (2003) 030; 05 (2003) 051.

[22] N. Demir and S. A. Bass, Phys. Rev. Lett. 102, 172302 (2009).

[23] V. Ozvenchuk, O. Linnyk, M. I. Gorenstein, E. L. Bratkovskaya, and W. Cassing, Phys. Rev. C 87, 064903 (2013).

[24] C. Sasaki and K.Redlich, Nucl. Phys. A832, 62 (2010).

[25] P. Deb, G. P. Kadam, and H. Mishra, Phys. Rev. D 94, 094002 (2016).

[26] A. S. Khvorostukhin, V. D. Toneev, and D. N. Voskresensky, Nucl. Phys. A915, 158 (2013).

[27] A. S. Khvorostukhin, V. D. Toneev, and D. N. Voskresensky, Nucl. Phys. A845, 106 (2010).

[28] H. B. Meyer, Phys. Rev. Lett. 100, 162001 (2008).

[29] K. Itakura, O. Morimatsu, and H. Otomo, Phys. Rev. D 77, 014014 (2008).

[30] R. Lang, N. Kaiser, and W. Weise, Eur. Phys. J. A 48, 109 (2012).

[31] D. Fernandiz-Fraile and A. Gomez Nicola, Eur. Phys. J. C 62, 37 (2009).

[32] S. Mitra, S. Ghosh, and S. Sarkar, Phys. Rev. C 85, 064917 (2012).

[33] M. Prakash, M. Prakash, R. Venugopalan, and G. Welke, Phys. Rep. 227, 321 (1993).

[34] A. Dobado and S. N. Santalla, Phys. Rev. D 65, 096011 (2002); A. Dobado and F. J. Llanes-Estrada, Phys. Rev. D 69, 116004 (2004).

[35] R. Marty, E. Bratkovskaya, W. Cassing, J. Aichelin, and H. Berrehrah, Phys. Rev. C 88, 045204 (2013). 
[36] A. Dobado, F. J. Llane-Estrada, and J. T. Rincon, Phys. Lett. B 702, 43 (2011).

[37] M. Bluhm, B. Kampfer, and K. Redlich, Phys. Rev. C 79, 055207 (2009).

[38] P. Chakraborty and J. I. Kapusta, Phys. Rev. C 83, 014906 (2011).

[39] K. Rajagopal and N. Trupuraneni, J. High Energy Phys. 03 (2010) 018; J. Bhatt, H. Mishra, and V. Sreekanth, J. High Energy Phys. 11 (2010) 106; Phys. Lett. B 704, 486 (2011); Nucl. Phys. A875, 181 (2012).

[40] S. Mitra and S. Sarkar, Phys. Rev. D 87, 094026 (2013); S. Mitra, S. Gangopadhyaya, and S. Sarkar, Phys. Rev. D 91, 094012 (2015).

[41] G. S. Denicol, H. Niemi, E. Molnar, and D. H. Rischke, Phys. Rev. D 85, 114047 (2012).

[42] M. Greif, F. Reining, I. Bouras, G. S. Denicol, Z. Xu, and C. Greiner, Phys. Rev. E 87, 033019 (2013).

[43] G. S. Denicol, H. Niemi, I. Bouras E. Molnar, Z. Xu, D. H. Rischke, and C. Greiner, Phys. Rev. D 89, 074005 (2014).

[44] J. I. Kapusta and J. M. Torres-Rincon, Phys. Rev. C 86, 054911 (2012).

[45] M. Iwasaki and T. Fukutome, J. Phys. G 36, 115012 (2009).

[46] S. Nam, Mod. Phys. Lett. A 30, 1550054 (2015).

[47] S. Ghosh, Int. J. Mod. Phys. E 24, 1550058 (2015).

[48] P. Singha, A. Abhishek, G. Kadam, S. Ghosh, and H. Mishra, arXiv:1705.03084v2.

[49] B. J. Schaefer, J. M. Pawlowski, and J. Wambach, Phys. Rev. D 76, 074023 (2007).
[50] U. S. Gupta and V. K. Tiwari, Phys. Rev. D 85, 014010 (2012).

[51] B. W. Mintz, R. Stiele, R. O. Ramos, and J. S. Bielich, Phys. Rev. D 87, 036004 (2013).

[52] S. Carignano, M. Buballa, and W. Elkamhawy, Phys. Rev. D 94, 034023 (2016).

[53] H. Mishra and R. K. Mohapatra, Phys. Rev. D 95, 094014 (2017).

[54] O. Scavenius, A. Mocsy, I. N. Mishustin, and D. H. Rischke, Phys. Rev. C 64, 045202 (2001).

[55] S. K. Ghosh, A. Lahiri, S. Majumder, M. G. Mustafa, S. Raha, and R. Ray, Phys. Rev. D 90, 054030 (2014).

[56] A. Bazavov et al., Phys. Rev. D 90,094503 (2014).

[57] M. Bluhm, B. Kampfer, and K. Redlich, Phys. Rev. C 84, 025201 (2011).

[58] M. Albright and J. I. Kapusta, Phys. Rev. C 93, 014903 (2016).

[59] P. Zhuang, J. Hufner, S. P. Klevansky, and L. Neise, Phys. Rev. D 51, 3728 (1995).

[60] F. Karsch, D. Kharzeev, and K. Tuchin, Phys. Lett. B 663, 217 (2008).

[61] S. Gavin, Nucl. Phys. A435, 826 (1985).

[62] A. Hosoya and K. Kajantie, Nucl. Phys. B250, 666 (1985).

[63] P. Danielewicz and M. Gyulassy, Phys. Rev. D 31, 53 (1985).

[64] D. T. Son and M. A. Stephanov, Phys. Rev. D 66, 076011 (2002); B. B. Brandt, A. Francis, H. B. Meyer, and D. Robaina, Phys. Rev. D 92, 094510 (2015); S. Gupta and R. Sharma arXiv:1710.05345. 